\title{
Homological stability for families of Coxeter groups
}

\author{
RICHARD HEPWORTH
}

\begin{abstract}
We prove that certain families of Coxeter groups and inclusions $W_{1} \hookrightarrow W_{2} \hookrightarrow \cdots$ satisfy homological stability, meaning that in each degree the homology $H_{*}\left(B W_{n}\right)$ is eventually independent of $n$. This gives a uniform treatment of homological stability for the families of Coxeter groups of type $A, B$ and $D$, recovering existing results in the first two cases, and giving a new result in the third. The key step in our proof is to show that a certain simplicial complex with $W_{n}$-action is highly connected. To do this we show that the barycentric subdivision is an instance of the "basic construction", and then use Davis's description of the basic construction as an increasing union of chambers to deduce the required connectivity.
\end{abstract}

20F55; $20 \mathrm{~J} 06$

\section{Introduction}

\subsection{Overview}

A family of groups $G_{1} \hookrightarrow G_{2} \hookrightarrow G_{3} \hookrightarrow \cdots$ is said to satisfy homological stability if the induced maps $H_{i}\left(B G_{n-1}\right) \rightarrow H_{i}\left(B G_{n}\right)$ are isomorphisms when $n$ is sufficiently large relative to $i$. Homological stability is known for many families of groups, including symmetric groups (see Nakaoka [23]), general linear groups (see Quillen [24]), mapping class groups of surfaces (see Harer [11]) and 3-manifolds (see Hatcher and Wahl [15]), diffeomorphism groups of highly connected manifolds (see Galatius and Randal-Williams [10]), and automorphism groups of free groups (see Hatcher [12] and Hatcher and Vogtmann [14]). Coxeter groups are abstract reflection groups, appearing in many areas of mathematics, such as root systems and Lie theory, geometric group theory, and combinatorics. See the books of Bourbaki [3], Davis [8] and Björner and Brenti [1] for introductions to Coxeter groups from each of these three viewpoints. In this paper we will show that homological stability holds for certain families of Coxeter groups.

Recall that a Coxeter matrix on a set $S$ is an $S \times S$ symmetric matrix $M$, with values in $\mathbb{N} \cup\{\infty\}$, satisfying $m_{s t}=1$ if $s=t$ and $m_{s t} \geqslant 2$ otherwise. The corresponding Coxeter group is the group generated by the elements of $S$, subject to the relations $(s t)^{m_{s t}}=e$ for $s, t \in S$. (When $m_{s t}=\infty$ no relation is imposed.) It is common to 
represent a Coxeter matrix by the equivalent Coxeter diagram. This is the graph with vertices $S$ and edges $\{s, t\}$ for $m_{s t} \geqslant 3$. The edge $\{s, t\}$ is labelled $m_{s t}$ if $m_{s t} \geqslant 4$. Now consider a sequence of finite Coxeter diagrams $\left(\Gamma_{n}\right)_{n \geqslant 1}$ of the form

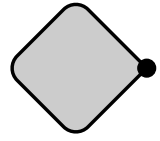

$\Gamma_{1}$

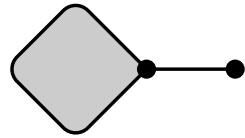

$\Gamma_{2}$

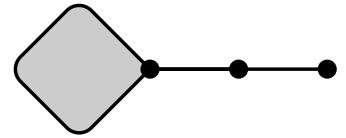

$\Gamma_{3}$

where every diagram has a preferred vertex, and each diagram is obtained from its predecessor by attaching a new preferred vertex to the old one by an unlabelled edge. Writing $W_{n}$ for the Coxeter group determined by $\Gamma_{n}$, the inclusion $\Gamma_{n-1} \hookrightarrow \Gamma_{n}$ induces an inclusion $W_{n-1} \hookrightarrow W_{n}$, and our main result states that the family

$$
W_{1} \hookrightarrow W_{2} \hookrightarrow W_{3} \hookrightarrow W_{4} \hookrightarrow \cdots
$$

satisfies homological stability.

Main Theorem The map $H_{*}\left(B W_{n-1}\right) \rightarrow H_{*}\left(B W_{n}\right)$ is an isomorphism in degrees satisfying $2 * \leqslant n$. Here homology is taken with arbitrary constant coefficients.

Observe that while the diagrams $\Gamma_{n}$ are assumed to be finite, it is not necessary for the groups $W_{n}$ to be finite.

\subsection{Homological stability for Coxeter groups of type $A, B$ and $D$}

The Main Theorem gives a uniform treatment of homological stability for the families of Coxeter groups of type $A_{n}, B_{n}$ and $D_{n}$. Recall that these are the Coxeter groups corresponding to the following diagrams, in which $n$ always denotes the total number of vertices:

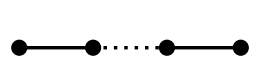

$A_{n}$

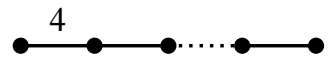

$B_{n}$

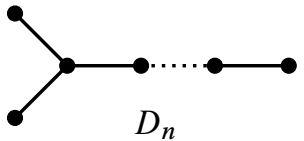

These families have an important place in the theory of Coxeter groups, since the classification of finite Coxeter groups states that a finite irreducible Coxeter group has type $A_{n}, B_{n}$ or $D_{n}$, or is dihedral, or is one of six exceptional examples. (See Appendix $C$ of [8].) The sequences $\left(A_{n}\right)_{n \geqslant 1},\left(B_{n+1}\right)_{n \geqslant 1}$ and $\left(D_{n+2}\right)_{n \geqslant 1}$ all have the form $\left(\Gamma_{n}\right)_{n \geqslant 1}$ described above, with the rightmost vertex taken as the preferred vertex, and therefore we may apply the main theorem to each one. In what follows we will use concrete descriptions of the groups of type $A_{n}, B_{n}$ and $D_{n}$ that can be found in Section 6.7 of [8]. 
1.2.1 Coxeter groups of type $\boldsymbol{A}$ For the sequence of diagrams $\left(A_{n}\right)_{n \geqslant 1}$, the corresponding sequence of Coxeter groups is

$$
\Sigma_{2} \hookrightarrow \Sigma_{3} \hookrightarrow \Sigma_{4} \hookrightarrow \Sigma_{5} \hookrightarrow \cdots
$$

where $\Sigma_{n}$ is the symmetric group on $n$ letters and the inclusions are given by extending permutations by the identity. Applying the Main Theorem, we recover the following classical result.

Corollary (Nakaoka) The map $H_{*}\left(B \Sigma_{n}\right) \rightarrow H_{*}\left(B \Sigma_{n+1}\right)$ is an isomorphism in degrees $2 * \leqslant n$. Here homology is taken with arbitrary constant coefficients.

In fact, Nakaoka computed $H_{*}\left(B \Sigma_{n} ; \mathbb{F}_{p}\right)$ for all primes $p$ in Theorem 6.3 of [23]. From this he deduced stability with $\mathbb{F}_{p}$ coefficients in Corollary 6.7 of [23]. The case of arbitrary coefficients follows. Nakaoka's computations can be used to show that $H_{k}\left(B \Sigma_{2 k-1} ; \mathbb{F}_{2}\right) \rightarrow H_{k}\left(B \Sigma_{2 k} ; \mathbb{F}_{2}\right)$ is not surjective for $k \geqslant 1$, so that the bound $2 * \leqslant n$ appearing in the corollary is sharp. Alternative proofs of Nakaoka stability, that do not rely on complete computations of $H_{*}\left(B \Sigma_{n} ; \mathbb{F}_{p}\right)$, can be found in the Ph D thesis of Maazen [21] and the papers of Kerz [19] and Randal-Williams [25].

1.2.2 Coxeter groups of type $\boldsymbol{B}$ For the sequence of diagrams $\left(B_{n+1}\right)_{n \geqslant 1}$, the corresponding sequence of Coxeter groups

$$
C_{2} \prec \Sigma_{2} \hookrightarrow C_{2} \prec \Sigma_{3} \hookrightarrow C_{2} \prec \Sigma_{4} \hookrightarrow C_{2} \prec \Sigma_{5} \hookrightarrow \cdots
$$

consists of the wreath products of the symmetric groups with the group $C_{2}$ of order 2 , and the inclusions are again given by extending permutations by the identity. Applying the Main Theorem gives the following result.

Corollary The map $H_{*}\left(B\left(C_{2} \backslash \Sigma_{n}\right)\right) \rightarrow H_{*}\left(B\left(C_{2} \backslash \Sigma_{n+1}\right)\right)$ is an isomorphism in degrees $2 * \leqslant n$. Here homology is taken with arbitrary constant coefficients.

This result can be found in a number of places in the literature. In particular, May computed $H_{*}\left(B\left(C_{2} \prec \Sigma_{n}\right) ; \mathbb{F}_{p}\right)$ for all $n \geqslant 1$ and all primes $p$. (See Cohen, Lada and May [7, Chapter I, Theorem 4.1] in the case $X=B C_{2} \sqcup\{*\}$.) From this computation one obtains the corollary above in the case of $\mathbb{F}_{p}$ coefficients, and the case of arbitrary coefficients follows. The corollary also follows from existing stability results such as Theorem A of [25] and Proposition 1.6 of Hatcher and Wahl's paper [15]. Observe that the bound $2 * \leqslant n$ is again sharp, since $C_{2} \prec \Sigma_{n}$ is a split extension of $\Sigma_{n}$. 
1.2.3 Coxeter groups of type $D$ For the sequence of diagrams $\left(D_{n+2}\right)_{n \geqslant 1}$, the corresponding sequence of Coxeter groups is

$$
H_{3} \hookrightarrow H_{4} \hookrightarrow H_{5} \hookrightarrow H_{6} \hookrightarrow \cdots
$$

where $H_{n}$ denotes the kernel of the homomorphism $C_{2}<\Sigma_{n} \rightarrow C_{2}$ that takes the product of the $C_{2}$-components. (We regard $C_{2}$ as the set $\{ \pm 1\}$ under multiplication.) The Main Theorem gives the following result.

Corollary Let $H_{n}$ denote the Coxeter group of type $D_{n}$. Then the inclusion map $H_{n+1} \hookrightarrow H_{n+2}$ induces an isomorphism $H_{*}\left(B H_{n+1}\right) \rightarrow H_{*}\left(B H_{n+2}\right)$ in degrees where $2 * \leqslant n$. Here homology is taken with arbitrary constant coefficients.

We believe that the result is new in the stated generality. However, Swenson [27] gave a generating set for the ring $H^{*}\left(B H_{n} ; \mathbb{F}_{2}\right)$, and deduced that the map $H^{k}\left(B H_{2 k+1} ; \mathbb{F}_{2}\right) \rightarrow$ $H^{k}\left(B H_{2 k} ; \mathbb{F}_{2}\right)$ is not surjective. (See Theorem 6.4.1 and the paragraph that follows it in [27].) It follows that $H_{k}\left(B H_{2 k} ; \mathbb{F}_{2}\right) \rightarrow H_{k}\left(B H_{2 k+1} ; \mathbb{F}_{2}\right)$ is not injective, so that the bound $2 * \leqslant n$ in the corollary is sharp.

\subsection{The superideal simplex reflection groups}

The Main Theorem applies to interesting families besides those of type $A_{n}, B_{n}$ and $D_{n}$ already considered. For example, if we fix an integer $m \geqslant 7$, then the main theorem shows that homological stability holds for the family of Coxeter groups associated to the sequence of diagrams $\left(\Gamma_{n}\right)_{n \geqslant 1}$

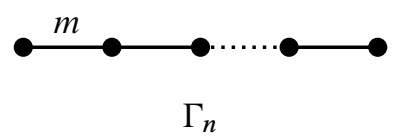

in which $\Gamma_{n}$ has a total of $(n+1)$ vertices, the rightmost one preferred. These are the superideal simplex reflection groups that appear in recent work of Calegari [6]. The first group is finite, while the rest are all infinite hyperbolic.

It is not difficult to construct other sequences of hyperbolic groups to which our main theorem applies. For example, we can construct sequences $\left(W_{n}\right)_{n \geqslant 1}$ in which the $W_{n}$ are all hyperbolic and have the same, arbitrary, virtual cohomological dimension (vcd). To do this we choose for $W_{1}$ an arbitrary hyperbolic right-angled Coxeter group with the desired vcd (see Januszkiewicz and Świątkowski [18]). Then by choosing a preferred element of the generating set of $W_{1}$ we extend to a sequence $\left(W_{n}\right)_{n \geqslant 1}$ of the kind appearing in the main theorem. By Moussong's condition [22, Theorem 17.1] (or see [8, Corollary 12.6.3]), the $W_{n}$ are all hyperbolic. By construction, the nerves $L_{n}$ of 
the $W_{n}$ satisfy $L_{n+1} \cong C L_{n}$. (See [8, Section 7.1] for the definition of the nerve.) Then by Davis's computation of the vcd of Coxeter groups [8, Corollary 8.5.5] they all have the same vcd.

\subsection{Homology of Coxeter groups in low degrees}

The Main Theorem was to some extent inspired by existing results on the homology of Coxeter groups in degree 1 and 2, as we now explain.

In degree 1 our main theorem states that the map $H_{1}\left(B W_{n-1}\right) \rightarrow H_{1}\left(B W_{n}\right)$ is an isomorphism for $n \geqslant 2$. This result has a simple proof. Let $W$ be a Coxeter group corresponding to Coxeter diagram $\Gamma$. Then one sees from the presentation of $W$ that the abelianization $W_{\mathrm{ab}}$ is naturally isomorphic to the elementary abelian 2-group on the path-components of the graph obtained from $\Gamma$ by deleting the edges with even or infinite label. In our situation $\Gamma_{n}$ is obtained from $\Gamma_{n-1}$ by attaching a single new vertex using an edge with label 3 , so that $\left(W_{n-1}\right)_{\mathrm{ab}} \rightarrow\left(W_{n}\right)_{\mathrm{ab}}$ is an isomorphism, and our stability result in degree 1 follows.

In degree 2 our main theorem states that the map $H_{2}\left(B W_{n-1}\right) \rightarrow H_{2}\left(B W_{n}\right)$ is an isomorphism for $n \geqslant 4$. The second homology groups $H_{2}(B W ; \mathbb{Z})$ of the finite Coxeter groups were computed by Ihara and Yokonuma in [17]. They showed that the result is an elementary abelian 2-group, and computed its rank. In particular, they observed that for the groups of type $A, B$ and $C$ the rank of $H_{2}(B W ; \mathbb{Z})$ stabilizes, and the stability range exactly corresponds to our result. Howlett [16] extended the work of Ihara and Yokonuma to arbitrary Coxeter groups. In our situation, his result shows that $H_{2}\left(B W_{n} ; \mathbb{Z}\right)$ is an elementary abelian 2-group whose rank is constant for $n \geqslant 3$, so that the isomorphism type of $H_{2}\left(B W_{n}\right)$ (now with arbitrary coefficients) is constant for $n \geqslant 3$. Thus Howlett's result almost implies our stability result in degree 2 , since it shows that the domain and range of the map in question are isomorphic.

\subsection{Outline of the proof of the main theorem}

The proof of the Main Theorem is modelled closely on existing techniques for proving Nakaoka's stability result for symmetric groups, which is the statement that the map $H_{*}\left(B \Sigma_{n}\right) \rightarrow H_{*}\left(B \Sigma_{n+1}\right)$ is an isomorphism for $2 * \leqslant n$. So we begin by explaining an approach to Nakaoka stability.

The proof of Nakaoka stability is by induction on $n$, the initial case $n=0$ being trivial. The inductive step uses the "complex of injective words", which we denote by $X$. This is the semisimplicial set whose $k$-simplices are ordered $(k+1)$-tuples in $\{1, \ldots, n+1\}$, with each element appearing at most once. It admits an action 
of $\Sigma_{n+1}$, and this action is transitive on $k$-simplices with stabilizer $\Sigma_{n-k-1}$. Moreover, the realization $\|X\|$ is $(n-1)$-connected. We now consider the spectral sequence arising from the filtration of $E W_{n} \times W_{n}\|X\|$ induced by the skeleta of $\|X\|$. Properties of the action of $\Sigma_{n+1}$ on $X$ allow us to identify the $E^{1}$-page of this spectral sequence in terms of the $H_{*}\left(B \Sigma_{n-k-1}\right)$ and the stabilization maps between them. The inductive hypothesis then allows us to compute the remaining pages of the spectral sequence in a range of degrees. The connectivity of $\|X\|$ guarantees that the sequence converges to $H_{*}\left(B \Sigma_{n}\right)$ in a range of degrees. From that point the result follows easily.

The hardest step here is the proof that $\|X\|$ is $(n-1)$-connected. There are several proofs of this in the literature; see Remark 39. The approach relevant to us is the following. Observe that $X$ is isomorphic to $\left(\Delta^{n}\right)^{\text {ord }}$, the semisimplicial set of simplices of $\Delta^{n}$ equipped with an ordering of their vertices. Now $\Delta^{n}$ is weakly Cohen-Macaulay of dimension $n$, meaning that it and the links of simplices within it satisfy certain connectivity bounds. A result of Randal-Williams [28, Proposition 7.9] states that if a complex $C$ is weakly Cohen-Macaulay of dimension $n$, then the realization $\left\|C^{\text {ord }}\right\|$ is $(n-1)$-connected. Applying this to $\Delta^{n}$, we obtain the connectivity of $\|X\|$.

Now here is a sketch of the proof of the main theorem. It follows the sketch proof of Nakaoka stability given above, and reduces to it in the case of Coxeter groups of type $A$.

(1) We construct a simplicial complex $\mathcal{C}^{n}$ with an action of $W_{n}$. For Coxeter groups of type $A$, the complex $\mathcal{C}^{n}$ is the $n$-simplex $\Delta^{n}$. We prove that $\mathcal{C}^{n}$ is weakly Cohen-Macaulay of dimension $n$.

(2) We form a semisimplicial set $\mathcal{D}^{n}$ with an action of $W_{n}$. For Coxeter groups of type $A$, this is the complex of injective words $X$. We show that $\mathcal{D}^{n}$ is the semisimplicial set of ordered simplices in $\mathcal{C}^{n}$ and conclude that it is $(n-1)-$ connected.

(3) Third, we use the spectral sequence associated to the filtration of $E W_{n} \times W_{n}\left\|\mathcal{D}^{n}\right\|$ induced by the skeleta of $\left\|\mathcal{D}^{n}\right\|$ to prove the theorem.

For Coxeter groups of type $A$, the proof that $\mathcal{C}^{n}$ is weakly Cohen-Macaulay of dimension $n$ is trivial. In general, we prove it as follows. We first prove that links of simplices in $\mathcal{C}^{n}$ are copies of $\mathcal{C}^{m}$ for appropriate $m<n$, so that the required connectivity bounds all follow if we can show that $\mathcal{C}^{n}$ is $(n-1)$-connected. To prove the latter, we make use of the "basic construction", a technique for constructing spaces with actions of Coxeter groups. (See [8, Chapters 5 and 8] and Section 2.5 below.) We identify the barycentric subdivision of $\mathcal{C}^{n}$ as an instance of the basic construction, and then use results of Davis on the topology of the basic construction (see Section 2.6) to conclude that it is $(n-1)$-connected. 
Outline of the paper In Section 2 we recall background material on Coxeter groups, then in Section 3 we establish some notation and discuss the groups of type $A, B$ and $D$ in detail. In Section 4 we study the subgroups $W_{i}$ of $W_{n}$, establishing important properties that will be used in the rest of the paper. Next, we move on to the simplicial complex $\mathcal{C}^{n}$ : in Section 5 we define it, in Section 6 we study the links of its simplices, and in Section 7 we show that $\left|\operatorname{sd} \mathcal{C}^{n}\right|$ is $(n-1)$-connected. Then we define $\mathcal{D}^{n}$ in Section 8, we show that it is isomorphic to the semisimplicial set of ordered simplices in $\mathcal{C}^{n}$, and conclude that it is $(n-1)$-connected. The proof of the Main Theorem is completed in Section 9.

Acknowledgements My thanks to Jarek Kędra, Ian Leary and Oscar Randal-Williams for helpful conversations as this work was being carried out, to Rachael Boyd for a careful reading of the paper, and to the referee for numerous helpful comments.

\section{Background on Coxeter groups}

Here we will recall some of the basic facts about the theory of Coxeter groups, giving references to [8] where possible. Hopefully this covers all of the material we will use in the rest of the paper. Alternative introductions to Coxeter groups are $[3 ; 1]$.

\subsection{Coxeter systems}

In Section 1.1 we defined Coxeter matrices, Coxeter diagrams, and the Coxeter group associated to a Coxeter matrix or diagram. A Coxeter system is a pair $(W, S)$ consisting of a group $W$ and a collection of involutions $S \subset W$ satisfying the following property: Let $\widetilde{W}$ denote the Coxeter group associated to the Coxeter matrix $M$ on $S$ defined by

$$
m_{s t}=\text { order of } s t .
$$

Then the homomorphism $\tilde{W} \rightarrow W$ extending the identity $S \rightarrow S$ is an isomorphism. See Section 3.3 of [8].

\subsection{Words}

Let $(W, S)$ be a Coxeter system. A word in $S$ is an ordered tuple $\left(t_{1}, \ldots, t_{r}\right)$ of elements of $S$. The word $\left(t_{1}, \ldots, t_{r}\right)$ has length $r$ and it represents the element $w=t_{1} \cdots t_{r}$ of $W$. Every element $w \in W$ is represented by some word, and its length $\ell(w)$ is the minimum length of a word representing it. A word is reduced if it has minimum length for the element of $W$ it represents. 


\subsection{The word problem}

An $M$-operation on a word in $S$ is a composite of the following elementary $M$ operations:

- Delete a subword $(s, s)$.

- Replace an alternating subword $(s, t, \ldots)$ of length $m(s, t)$ with the subword $(t, s, \ldots)$ of the same length.

Observe that these operations do not alter the element of $W$ represented by the word, since all elements of $S$ are involutions, and since the relation $(s t)^{m(s, t)}=e$ can be rewritten as $(s t \cdots)=(t s \cdots)$, where each side is an alternating word of length $m(s, t)$. Observe also that these operations either preserve or reduce the length of a word. Tits' solution to the word problem in Coxeter groups states that a word is reduced if and only if it cannot be shortened by an $M$-operation, and that two reduced words represent the same element if and only if they are related by a sequence of elementary $M$-operations of the second kind. See Section 3.4 of [8].

\subsection{Special subgroups}

Let $(W, S)$ be a Coxeter system. Given $T \subset S$, we denote by $W_{T}$ the subgroup of $W$ generated by $T$, and we refer to $W_{T}$ as a special subgroup of $W$. Then $\left(W_{T}, T\right)$ is again a Coxeter system. (This is why our sequence of homomorphisms $W_{1} \rightarrow W_{2} \rightarrow \cdots$ is in fact a sequence of inclusions.) See Section 4.1 of [8].

Given $T, U \subset S$ we say that $w \in W$ is $(T, U)$ reduced if it cannot be represented by a reduced word starting with an element of $T$ or ending with an element of $U$. If $x$ is $(T, U)$ reduced then a result of Kilmoyer, Solomon and Tits shows that $W_{T} \cap x W_{U} x^{-1}=W_{V}$, where $V=T \cap x U x^{-1}$. See Lemma 2 of [26]. In particular, this shows that if $T, U \subset S$ then $W_{T} \cap W_{U}=W_{T \cap U}$. See Theorem 4.1.6 of [8] for a proof of this special case.

\subsection{The basic construction}

The "basic construction" is a method for building spaces with an action of a Coxeter group. It can be used, for example, to study the topology of the Coxeter complex and Davis complex of a Coxeter group. (Our discussion is tailored to the case of Coxeter groups. For an approach to the basic construction that applies to more general groups see Chapter II.12 of [5].)

Let $(W, S)$ be a Coxeter system. A mirrored space over $S$ is a space $X$ together with subspaces $X_{s} \subset X$, called mirrors, one for each $s \in S$. We assume that $X$ is 
a CW-complex and that the mirrors are subcomplexes. The basic construction is the space

$$
\mathcal{U}(W, X)=(W \times X) / \sim,
$$

where $(v, x) \sim(w, y)$ if and only if $x=y$ and $v^{-1} w$ belongs to the subgroup generated by the $s \in S$ for which $x \in X_{S}$. The basic construction is equipped with the action of $W$ by left translation, and we identify $X$ with the image of $\{e\} \times X$ in $\mathcal{U}(W, X)$. Observe that $\mathcal{U}(W, X)$ has the structure of a CW-complex in which each translate $w X$ is a subcomplex. See Section 5.1 of [8].

\subsection{The increasing union of chambers}

For us the most important feature of the basic construction is that it can be described as an increasing union of chambers, meaning copies of $X$, as we now recall from Section 8.1 of [8]. Given $w \in W$, let

$$
\operatorname{In}(w)=\{s \in S \mid \ell(w s)<\ell(w)\}
$$

denote the set of letters with which a reduced expression for $w$ can end, and let

$$
X^{\operatorname{In}(w)}=\bigcup_{s \in \operatorname{In}(w)} X_{S}
$$

denote the corresponding union of mirrors. Order the elements of $W$ as $w_{0}, w_{1}, w_{2}, \ldots$, where $w_{0}=e$ and $\ell\left(w_{m}\right) \leqslant \ell\left(w_{m+1}\right)$ for $m \geqslant 0$. Define

$$
P_{m}=\bigcup_{i=0}^{m} w_{i} X,
$$

so that $\mathcal{U}(W, X)$ is the increasing union of the subcomplexes $P_{m}$. Then

$$
P_{m}=P_{m-1} \cup w_{m} X \quad \text { and } \quad P_{m-1} \cap w_{m} X=w_{m} X^{\operatorname{In}\left(w_{m}\right)} .
$$

The latter equation is by Lemma 8.1.1 of [8]. It will be useful to us since it specifies exactly how each chamber is attached to its predecessor, so that we can study the topology of $\mathcal{U}(W, X)$ inductively by adding one chamber at a time.

\section{Notation and examples}

In this section we establish some notation that will be used throughout the rest of the paper. We also establish in more detail the Coxeter groups of type $A, B$ and $D$, which will be used for illustration throughout the rest of the paper. Fix a sequence $\left(\Gamma_{n}\right)_{n \geqslant 1}$ of the kind described in the introduction. 
Definition 1 (the elements $s_{1}, \ldots, s_{n}$ ) For $n \geqslant 1$ we define $s_{n}$ to be the preferred vertex of $\Gamma_{n}$, as in the following diagram:

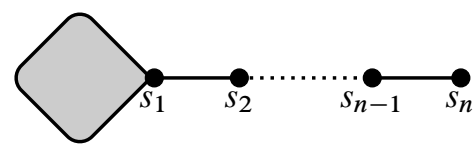

Thus the special subgroup of $W_{n}$ generated by $s_{1}, \ldots, s_{n}$ is a copy of the Coxeter group of type $A_{n}$, and so is isomorphic to $\Sigma_{n+1}$. See Example 2 below.

Example 2 (groups of type $A$ ) Consider the sequence of diagrams $\left(A_{n}\right)_{n \geqslant 1}$ :

$A_{n}$

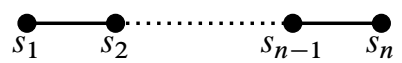

In this case the group $W_{n}$ may be identified with $\Sigma_{n+1}$, the symmetric group on letters $1, \ldots,(n+1)$, where $s_{i}$ is the adjacent transposition $(i i+1)$. See Example 6.7.1 of [8].

Example 3 (groups of type $B$ ) Consider the sequence of diagrams $\left(B_{n+1}\right)_{n \geqslant 1}$ :

$$
B_{n+1}
$$

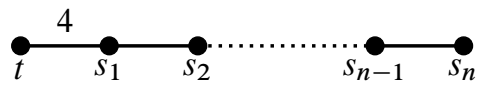

The group $W_{n}$ may be identified with the wreath product $C_{2} \prec \Sigma_{n+1}$, where $t$ is identified with the generator of $C_{2}$ and $s_{i}$ is again identified with the adjacent transposition $(i i+1)$. For concreteness, we further identify $C_{2}<\Sigma_{n+1}$ with the set of permutations $\sigma$ of $\{ \pm 1, \ldots, \pm(n+1)\}$ that satisfy $\sigma(-i)=-\sigma(i)$ for all $i$. In this setting $t$ is the permutation that sends \pm 1 to $\mp 1$ and fixes all other elements, while $s_{i}$ is the permutation that sends $\pm i$ to $\pm(i+1)$ and vice versa and fixes all other elements. See Example 6.7.2 of [8].

Example 4 (groups of type $D$ ) Consider the sequence of diagrams $\left(D_{n+2}\right)_{n \geqslant 1}$ :

$$
D_{n+2}
$$

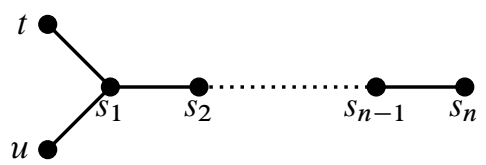

The group $W_{n}$ may be identified as the kernel of the homomorphism $C_{2}<\Sigma_{n+2} \rightarrow C_{2}$ that takes the product of the $C_{2}$-components. Regarding $C_{2} 2 \Sigma_{n+2}$ as a group of permutations of $\{ \pm 1, \ldots, \pm(n+2)\}$, this kernel consists of the permutations that send an even number of the positive elements to negative ones. Under this identification, $t$ corresponds to the element that negates \pm 1 and \pm 2 and fixes all other elements; 
$u$ transposes 1 and $2,-1$ and -2 , and fixes all other elements; and $s_{i}$ sends $\pm(i+1)$ to $\pm(i+2)$ and vice versa and fixes all other elements. See Example 6.7.3 of [8].

Definition 5 (the additional diagrams $\Gamma_{0}$ and $\Gamma_{-1}$ ) We extend the sequence $\left(\Gamma_{n}\right)_{n \geqslant 1}$ to the left by two terms as follows. Define $\Gamma_{0}$ to be the diagram obtained from $\Gamma_{1}$ by deleting the preferred vertex, and define $\Gamma_{-1}$ to be the diagram obtained from $\Gamma_{1}$ by deleting the preferred vertex and all vertices that shared an edge with it.

Example 6 ( $\Gamma_{0}$ and $\Gamma_{-1}$ for Coxeter groups of type $A, B$ and $\left.D\right)$ For the sequence $\left(A_{n}\right)_{n \geqslant 1}$, the diagrams $A_{0}$ and $A_{-1}$ are both empty. For $\left(B_{n+1}\right)_{n \geqslant 1}$, the diagram $B_{0+1}$ consists of the single vertex $t$ and $B_{-1+1}$ is empty. For $\left(D_{n+2}\right)_{n \geqslant 1}$, the diagram $D_{0+2}$ consists of the two vertices $t$ and $u$ with no edge, and $D_{-1+2}$ is empty.

Definition 7 (the generating sets $S_{n}$ ) Let $\left(\Gamma_{n}\right)_{n \geqslant 1}$ be a sequence of the kind described in the introduction, and let $\left(\Gamma_{n}\right)_{n \geqslant-1}$ be the extension just described. Then for $n \geqslant-1$ we define $S_{n}$ to be the set of vertices of $\Gamma_{n}$. Thus $\left(W_{n}, S_{n}\right)$ is a Coxeter system for each $n \geqslant-1$.

\section{The subgroups $W_{-1} \subset W_{0} \subset W_{1} \subset \cdots \subset W_{n}$}

From this point onwards, unless stated otherwise, we fix a sequence $\left(\Gamma_{n}\right)_{n \geqslant 1}$ and an integer $n \geqslant 1$.

This section will study the sequence of subgroups $W_{-1} \subset W_{0} \subset \cdots \subset W_{n}$, and in particular the cosets of $W_{k}$ in $W_{n}$ for $k<n$. We do this now because the geometric objects that will appear later in the paper are constructed by considering these cosets. Throughout the section we will illustrate the results using the sequences $\left(A_{n}\right)_{n \geqslant 1}$, $\left(B_{n+1}\right)_{n \geqslant 1}$ and $\left(D_{n+2}\right)_{n \geqslant 1}$ that were explained in Examples 2, 3 and 4 . The key idea to bear in mind is that $W_{n} / W_{n-1}$ is "the natural set for $W_{n}$ to act on". For example, for groups of type $A$, where $W_{n}=\Sigma_{n+1}$, we will see that $W_{n} / W_{n-1}$ is isomorphic to $\{1, \ldots, n+1\}$ with the permutation action.

Proposition 8 (left cosets of $W_{n-1}$ ) (1) Let $i$ lie in the range $1 \leqslant i \leqslant n$. Then left-multiplication by the element $s_{i}$ fixes the set

$$
\left\{s_{1} \cdots s_{n} W_{n-1}, s_{2} \cdots s_{n} W_{n-1}, \ldots, s_{n} W_{n-1}, W_{n-1}\right\} .
$$

It acts on the set by transposing $s_{i} \cdots s_{n} W_{n-1}$ and $s_{i+1} \cdots s_{n} W_{n-1}$, and fixing the remaining elements. Here the product $\left(s_{i+1} \cdots s_{n}\right)$ is omitted when $i=n$. 
(2) For $c \in W_{n}$ the cosets

$$
c\left(s_{1} \cdots s_{n}\right) W_{n-1}, \quad \ldots, \quad c s_{n} W_{n-1}, \quad c W_{n-1}
$$

are pairwise distinct.

Proof One can verify the identities

$$
\begin{aligned}
s_{i}\left(s_{j} \cdots s_{n}\right) & =\left(s_{j} \cdots s_{n}\right) s_{i} \quad \text { for } i<j-1, \\
s_{i}\left(s_{i+1} \cdots s_{n}\right) & =s_{i} \cdots s_{n}, \\
s_{i}\left(s_{i} \cdots s_{n}\right) & =s_{i+1} \cdots s_{n}, \\
s_{i}\left(s_{j} \cdots s_{n}\right) & =\left(s_{j} \cdots s_{n}\right) s_{i-1} \quad \text { for } i>j,
\end{aligned}
$$

and then the first part follows immediately. (The product $s_{i+1} \cdots s_{n}$ is omitted when $i=n$.) For the second part, if $c s_{j} \cdots s_{n} W_{n-1}=c s_{k} \cdots s_{n} W_{n-1}$ with $j<k$, then $\left(s_{n} \cdots s_{j}\right)\left(s_{k} \cdots s_{n}\right) \in W_{n-1}$. But

$$
\left(s_{n} \cdots s_{j}\right)\left(s_{k} \cdots s_{n}\right)=\left(s_{k-1} \cdots s_{n} \cdots s_{k-1}\right)\left(s_{k-2} \cdots s_{j}\right),
$$

where the second factor on the right is omitted in the case $j=k-1$. This implies that $s_{n} \in W_{n-1}$, which is a contradiction.

Example $9\left(W_{n} / W_{n-1}\right.$ for groups of type $A, B$ and $\left.D\right)$ We illustrate Proposition 8 for the sequence $\left(A_{n}\right)_{n \geqslant 1}$ here. As explained in Example 2, we may regard $W_{n}$ as the group $\Sigma_{n+1}$ of permutations of the set $\{1, \ldots, n+1\}$. This allows us to identify $W_{n} / W_{n-1}$ via the isomorphism

$$
W_{n} / W_{n-1} \stackrel{\cong}{\longrightarrow}\{1, \ldots, n+1\}, \quad \sigma W_{n-1} \longmapsto \sigma(n+1),
$$

which respects the $W_{n}$ action on each side, and which maps the coset $s_{i} \cdots s_{n} W_{n-1}$ to the letter $i$. (When $i=n+1$ the product $s_{i} \cdots s_{n}$ is omitted.) So for these groups, the first part of the proposition above amounts to the fact that $s_{i}$ transposes the elements $i$ and $i+1$. For $\sigma \in W_{n}$, the cosets

$$
\sigma\left(s_{1} \cdots s_{n}\right) W_{n-1}, \quad \ldots, \quad \sigma s_{n} W_{n-1}, \quad \sigma W_{n-1}
$$

correspond under the isomorphism above to the elements $\sigma(1), \ldots, \sigma(n+1)$. So the second part of the proposition amounts to the fact that these elements are distinct since $\sigma$ is a permutation.

A similar account can be given for the sequences $\left(B_{n+1}\right)_{n \geqslant 1}$ and $\left(D_{n+2}\right)_{n \geqslant 1}$, this time using Examples 3 and 4. For $\left(B_{n+1}\right)_{n \geqslant 1}$ the account is identical after replacing $\{1, \ldots, n+1\}$ with $\{ \pm 1, \ldots, \pm(n+1)\}$. For $\left(D_{n+2}\right)_{n \geqslant 1}$ the set being acted on is now $\{ \pm 1, \ldots, \pm(n+2)\}$, and the isomorphism sends $\sigma W_{n}$ to $\sigma(n+2)$. 
Proposition $10 W_{i-1} \cap\left(s_{i} \cdots s_{n} W_{n-1} s_{n} \cdots s_{i}\right)=W_{i-2}$ for $1 \leqslant i \leqslant n$.

Proof The element $s_{i} \cdots s_{n}$ is $\left(W_{i-1}, W_{n-1}\right)$-reduced, meaning that it does not have a reduced representative beginning with an element of $W_{i-1}$ or ending with a representative of $W_{n-1}$. Thus, as we recalled in Section 2.4, the intersection

$$
W_{i-1} \cap s_{i} \cdots s_{n} W_{n-1} s_{n} \cdots s_{i}
$$

is the subgroup generated by $T=S_{i-1} \cap\left(s_{i} \cdots s_{n} S_{n-1} s_{n} \cdots s_{i}\right)$. So it will be enough to show that $T=S_{i-2}$. If $j \leqslant i-2$ then $s_{n} \cdots s_{i} s_{j} s_{i} \cdots s_{n}=s_{j}$, and consequently $S_{i-2} \subset T$. So suppose that $t \in T \backslash S_{i-2}$. Thus $t \in S_{i-1} \backslash S_{i-2}$ and $s_{n} \cdots s_{i} t s_{i} \cdots s_{n} \in$ $S_{n-1}$. By the first condition we have $m_{s_{i} t} \geqslant 3$. By the second condition the word $\left(s_{n}, \ldots, s_{i}, t, s_{i}, \ldots, s_{n}\right)$ represents an element of $S_{n-1}$, so is not reduced. By the solution to the word problem recalled in Section 2.3, we must therefore be able to apply an $M$-operation to this word, but by inspection this is only possible if $m_{s_{i}}$ is exactly 3 . But in that case $\left(s_{n}, \ldots, s_{i}, t, s_{i}, \ldots, s_{n}\right)$ is already reduced, contradicting the second condition (see Sections 2.2 and 2.3).

Example 11 In the case of the sequence $\left(A_{n}\right)_{n \geqslant 1}$, the previous proposition can be explained as follows. The group $W_{n}$ is identified with the symmetric group $\Sigma_{n+1}$ on the set $\{1, \ldots, n+1\}$, and $W_{k-1}$ is the subgroup that fixes $(k+1), \ldots,(n+1)$. Thus:

- $W_{i-1}$ is the subgroup that fixes $(i+1), \ldots,(n+1)$.

- $s_{i} \cdots s_{n} W_{n-1} s_{n} \cdots s_{i}$ is the subgroup that fixes $i$.

- $W_{i-2}$ is the subgroup that fixes $i, \ldots,(n+1)$.

This makes the proposition's claim that $W_{i-1} \cap\left(s_{i} \cdots s_{n} W_{n-1} s_{n} \cdots s_{i}\right)=W_{i-1}$ immediate. For the sequences $\left(B_{n+1}\right)_{n \geqslant 1}$ and $\left(D_{n+2}\right)_{n \geqslant 1}$ one can give a similar account.

Proposition 12 Let $i$ lie in the range $1 \leqslant i \leqslant n$. If $\sigma, \tau \in W_{n}$ satisfy

$$
\sigma s_{j} \ldots s_{n} W_{n-1}=\tau s_{j} \ldots s_{n} W_{n-1} \quad \text { for } j=i, \ldots, n+1,
$$

then $\sigma^{-1} \tau \in W_{i-2}$. Here the product $\left(s_{j} \cdots s_{n}\right)$ is omitted when $j=n+1$.

Proof The proposition is equivalent to the claim that

$$
W_{n-1} \cap\left(s_{n} W_{n-1} s_{n}\right) \cap \cdots \cap\left(s_{i} \cdots s_{n} W_{n-1} s_{n} \cdots s_{i}\right)=W_{i-2},
$$

which is proved by downward induction on $i$. The initial case $i=n+1$ is immediate, and the induction step follows from Proposition 10. 


\section{The simplicial complex $\mathcal{C}^{n}$}

Now we introduce the simplicial complex $\mathcal{C}^{n}$ that will be central to our proof of the Main Theorem. The definition of $\mathcal{C}^{n}$ is motivated by the case of Coxeter groups of type $A$, where $W_{n}$ is the symmetric group $\Sigma_{n+1}$, and $\mathcal{C}^{n}$ is nothing other than the $n$-simplex. As explained in Section 1.5, this is relevant since the semisimplicial set of ordered simplices in the $n$-simplex is the "complex of injective words", which appears in several existing proofs of homological stability for the symmetric groups. In the general case, $\mathcal{C}^{n}$ is designed so that its semisimplicial set of ordered simplices can play the role of the complex of injective words in a proof of homological stability for the sequence $\left(W_{n}\right)_{n \geqslant 1}$.

The main result of this section is that $\mathcal{C}^{n}$ is weakly Cohen-Macaulay of dimension $n$. The proof relies on propositions that will be established in the following two sections.

Definition 13 (the simplicial complex $\mathcal{C}^{n}$ ) Given $n \geqslant 0$, we define $\mathcal{C}^{n}$ to be the $n$-dimensional simplicial complex with vertex set $W_{n} / W_{n-1}$ and with $k$-simplices given by the subsets

$$
C=\left\{c\left(s_{n-k+1} \cdots s_{n}\right) W_{n-1}, \ldots, c s_{n} W_{n-1}, c W_{n-1}\right\}
$$

for $0 \leqslant k \leqslant n$ and $c \in W_{n}$. Proposition 8 shows that $C$ does indeed have cardinality $(k+1)$. In this situation we call $c$ a lift of the simplex $C$.

Remark 14 We chose the name "lift" to emphasize the formal similarity with the concept of the same name that appears in Definition 2.1 of Wahl [28].

A given simplex can have many lifts. Choosing a lift for a simplex induces an ordering of its vertices, and all orderings occur in this way. For if $c$ lifts a $k$-simplex $C$ then so does $c s_{n-k+i+1}$, and the induced orderings differ by transposition of the $i^{\text {th }}$ and $(i+1)^{\text {st }}$ vertices (see Proposition 8). This makes it simple to verify that $\mathcal{C}^{n}$ is indeed a simplicial complex, for if $C$ is a simplex of $\mathcal{C}^{n}$ and $D \subset C$ is a nonempty subset, then we may choose a lift $c$ of $C$ such that $D$ is a terminal segment in the induced ordering. Then $c$ is also a lift of $D$.

The natural action of $W_{n}$ on $W_{n} / W_{n-1}$ extends to an action on $\mathcal{C}^{n}$. For if $C$ is a simplex of $\mathcal{C}^{n}$ with lift $c$, and if $w \in W_{n}$, then $w C$ is a simplex of $\mathcal{C}^{n}$ with lift $w c$.

We now give a concrete description of $\mathcal{C}^{n}$ for the families of Coxeter groups of type $A$, $B$ and $D$. See Examples 2, 3 and 4 for the description of these groups, and Example 9 for a description of $W_{n} / W_{n-1}$ in each case. 
Example $15\left(\mathcal{C}^{n}\right.$ for groups of type $\left.A\right)$ For the sequence of diagrams $\left(A_{n}\right)_{n \geqslant 1}$ we saw in Example 2 that $W_{n}=\Sigma_{n+1}$ is the symmetric group on $(n+1)$ letters. Then $\mathcal{C}^{n}$ is the $n$-dimensional simplex $\Delta^{n}$ with the action of $\Sigma_{n+1}$ that permutes the vertices. For as in Example 9 the vertex set $W_{n} / W_{n-1}$ of $\mathcal{C}^{n}$ is isomorphic to $\{1, \ldots, n+1\}$ via the map that sends $\sigma W_{n-1}$ to $\sigma(n+1)$. Under this isomorphism, an element $\sigma \in W_{n}$ is a lift of the $k$-simplex

$$
C=\{\sigma(n-k+1), \ldots, \sigma(n+1)\},
$$

and every subset of $\{1, \ldots, n+1\}$ arises in this way.

Example 16 ( $\mathcal{C}^{n}$ for groups of type $B$ ) For the sequence of diagrams $\left(B_{n+1}\right)_{n \geqslant 1}$ we saw in Example 3 that $W_{n}$ is the group of permutations $\sigma$ of the set $\{ \pm 1, \ldots, \pm(n+1)\}$ satisfying the rule $\sigma(-i)=-\sigma(i)$ for all $i$. In this case $\mathcal{C}^{n}$ is isomorphic to the hyperoctahedron of dimension $n$, which is the simplicial complex whose vertex set is $\{ \pm 1, \ldots, \pm(n+1)\}$ and whose simplices are the subsets containing at most one element from each pair $\{i,-i\}$. In particular, its realization is homeomorphic to the $n$-sphere. To obtain this description, we use the isomorphism $W_{n} / W_{n-1} \rightarrow$ $\{ \pm 1, \ldots, \pm(n+1)\}$ sending $\sigma W_{n-1}$ to $\sigma(n+1)$, as in Example 9. Under this isomorphism an element $\sigma$ lifts the $k$-simplex

$$
C=\{\sigma(n-k+1), \ldots, \sigma(n+1)\},
$$

so that a subset of $\{ \pm 1, \ldots, \pm(n+1)\}$ spans a simplex of $\mathcal{C}^{n}$ if and only if it does not contain any element and its negative.

Example 17 ( $\mathcal{C}^{n}$ for groups of type $\left.D\right)$ For the sequence of diagrams $\left(D_{n+2}\right)_{n \geqslant 1}$ we saw in Example 4 that $W_{n}$ is the group of permutations of $\{ \pm 1, \ldots, \pm(n+2)\}$ that satisfy the rule $\sigma(-i)=-\sigma(i)$ and that send an even number of the positive elements to negative ones. In this case $\mathcal{C}^{n}$ is the $n$-skeleton of the $(n+1)$-dimensional hyperoctahedron. In other words, it is the simplicial complex with vertex set $\{ \pm 1, \ldots, \pm(n+2)\}$, and whose simplices are the subsets of size at most $n+1$ containing at most one element from each pair $\{i,-i\}$. (Compare with Example 16.) In particular, the realization of $\mathcal{C}^{n}$ has the homotopy type of the wedge of $\left(2^{n}-1\right)$ copies of the $n$-dimensional sphere. To obtain this description recall from Example 9 that the vertex set $W_{n} / W_{n-1}$ is identified with $\{ \pm 1, \ldots, \pm(n+2)\}$ via the map sending $\sigma W_{n-1}$ to $\sigma(n+2)$. Under this identification the $k$-simplex with lift $\sigma$ is

$$
C=\{\sigma(n-k+2), \ldots, \sigma(n+2)\},
$$

so that a subset of $\{ \pm 1, \ldots, \pm(n+2)\}$ spans a simplex if and only if it does not contain any element and its negative and has size at most $n$. 
Recall from Definition 3.4 of [15] that a simplicial complex is called weakly CohenMacaulay of dimension $n$ if it is $(n-1)$-connected and the link of each $p$-simplex is $(n-p-2)$-connected. In each of the three examples above, $\mathcal{C}^{n}$ has the homotopy type of a wedge of $n$-dimensional spheres, and so is $(n-1)$-connected. In fact, it is not hard to see that in these examples $\mathcal{C}^{n}$ is weakly Cohen-Macaulay of dimension $n$. This is an instance of the following general fact.

Theorem $18 \mathcal{C}^{n}$ is weakly Cohen-Macaulay of dimension $n$.

The proof of this theorem relies on Propositions 19, 26 and 27, which are proved over the course of the next two sections.

Proof By Proposition 19, if $C$ is a $p$-simplex of $\mathcal{C}^{n}$ then $\operatorname{lk}_{\mathcal{C}^{n}}(C) \cong \mathcal{C}^{n-p-1}$. It therefore suffices to show that $\mathcal{C}^{n}$ is $(n-1)$-connected for all $n$, or equivalently that the barycentric subdivision $\operatorname{sd}^{n}$ is $(n-1)$-connected for all $n$. Now Proposition 26 shows that $\left|\operatorname{sd} \mathcal{C}^{n}\right|$ is homeomorphic to the basic construction $\mathcal{U}\left(W_{n},|\Delta|\right)$, while Proposition 27 shows that $\mathcal{U}\left(W_{n},|\Delta|\right)$ is $(n-1)$-connected.

\section{Links of simplices of $\mathcal{C}^{n}$}

The aim of this section is to prove the following proposition, which was used in the proof of Theorem 18 above.

Proposition 19 Let $C$ be a $p$-simplex of $\mathcal{C}^{n}$. Then $\operatorname{lk}_{\mathcal{C}^{n}}(C) \cong \mathcal{C}^{n-p-1}$.

In the next section we prove that $\mathcal{C}^{n}$ is $(n-1)$-connected. This, combined with the proposition above, shows that the links of $p$-simplices in $\mathcal{C}^{n}$ are $(n-p-2)$-connected, and consequently that $\mathcal{C}^{n}$ is weakly Cohen-Macaulay of dimension $n$.

Example 20 (links in $\mathcal{C}^{n}$ for groups of type $A, B$ and $D$ ) In Examples 15, 16 and 17 we gave concrete descriptions of $\mathcal{C}^{n}$ for each of the sequences $\left(A_{n}\right)_{n \geqslant 1},\left(B_{n+1}\right)_{n \geqslant 1}$ and $\left(D_{n+2}\right)_{n \geqslant 1}$. These descriptions can be used to illustrate Proposition 19. For example, if we take the sequence $\left(B_{n+1}\right)_{n \geqslant 1}$, then $\mathcal{C}^{n}$ is the hyperoctahedron of dimension $n$, ie, the simplicial complex with vertices $\{ \pm 1, \ldots, \pm(n+1)\}$ in which a subset of the vertices spans a simplex if and only if it does not contain any element and its negative. Thus $\mathcal{C}^{2}, \mathcal{C}^{1}$ and $\mathcal{C}^{0}$ are as shown in Figure 1 (in $\mathcal{C}^{1}$ and $\mathcal{C}^{0}$ the dashed parts are not included). We see that in $\mathcal{C}^{2}$ the link of the vertex $\{3\}$ is a copy of $\mathcal{C}^{1}$, while the link of the edge $\{2,3\}$ is a copy of $\mathcal{C}^{0}$. 

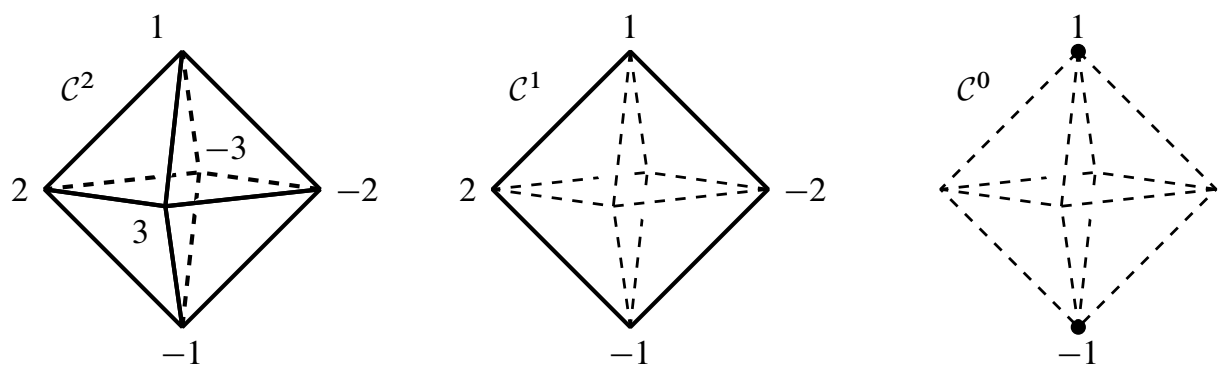

Figure 1: The hyperoctohedra $\mathcal{C}^{2}, \mathcal{C}^{1}, \mathcal{C}^{0}$

Proof of Proposition 19 Choose a lift $c$ of $C$. Define

$$
\phi: W_{n-p-1} / W_{n-p-2} \rightarrow W_{n} / W_{n-1}
$$

by $\phi\left(d W_{n-p-2}\right)=c d s_{n-p} \cdots s_{n} W_{n-1}$ for $d \in W_{n-p-1}$. This is well defined since every generator of $W_{n-p-2}$ commutes with $s_{n-p}, \ldots, s_{n}$. Observe that the domain and range of $\phi$ are the vertex sets of $\mathcal{C}^{n-p-1}$ and $\mathcal{C}^{n}$ respectively.

Claim 1 The map $\phi$ is an injection.

To prove this claim, let $d, d^{\prime} \in W_{n-p-1}$ satisfy

$$
c d\left(s_{n-p} \cdots s_{n}\right) W_{n-1}=c d^{\prime} s_{n-p} \cdots s_{n} W_{n-1} .
$$

Then

$$
d^{-1} d^{\prime} \in W_{n-p-1} \cap\left(s_{n-p} \cdots s_{n}\right) W_{n-1}\left(s_{n} \cdots s_{n-p}\right)=W_{n-p-2},
$$

the latter equation by Proposition 10. Thus $d^{\prime} W_{n-p-2}=d W_{n-p-2}$.

Claim 2 The map $\phi$ sends simplices of $\mathcal{C}^{n-p-1}$ to simplices of $\operatorname{lk}_{\mathcal{C}^{n}}(C)$.

To prove this, suppose that $D$ is an $i$-simplex of $\mathcal{C}^{n-p-1}$. Let $d \in W_{n-p-1}$ be a lift of $D$. Then

$$
\phi D=\left\{c d s_{n-p-i} \cdots s_{n} W_{n-1}, \ldots, c d s_{n-p} \cdots s_{n} W_{n-1}\right\}
$$

while

$$
\begin{aligned}
C & =\left\{c s_{n-p+1} \cdots s_{n} W_{n-1}, \ldots, c s_{n} W_{n-1}, c W_{n-1}\right\}, \\
& =\left\{c d s_{n-p+1} \cdots s_{n} W_{n-1}, \ldots, c d s_{n} W_{n-1}, c d W_{n-1}\right\} .
\end{aligned}
$$

Thus $\phi D \cap C=\varnothing$ by Proposition 8 , and $\phi D \cup C$ is a simplex of $\mathcal{C}^{n}$ with lift $c d$, so that $\phi D$ is a simplex of $\operatorname{lk}_{\mathcal{C}^{n}}(C)$ as claimed. 
Claim 3 Every simplex of $\operatorname{lk}_{\mathcal{C}^{n}}(C)$ has the form $\phi D$ for some simplex $D$ of $\mathcal{C}^{n-p-1}$.

To prove this, suppose that $\bar{D}$ is an $i-\operatorname{simplex}$ of $\operatorname{lk}_{\mathcal{C}^{n}}(C)$. Then $\bar{D} \cap C=\varnothing$ and $\bar{D} \cup C$ is a simplex of $\mathcal{C}^{n}$. Let $c^{\prime}$ be a lift of $\bar{D} \cup C$, and assume without loss that the ordering it induces on $\bar{D} \cup C$ contains $\bar{D}$ as an initial segment and $C$ as a terminal segment with the ordering induced by $c$. Thus

$$
\bar{D}=\left\{c^{\prime}\left(s_{n-p-i} \cdots s_{n}\right) W_{n-1}, \ldots, c^{\prime}\left(s_{n-p} \cdots s_{n}\right) W_{n-1}\right\}
$$

and

$$
c^{\prime}\left(s_{n-p+k} \cdots s_{n}\right) W_{n-1}=c\left(s_{n-p+k} \cdots s_{n}\right) W_{n-1}
$$

for $k=1, \ldots, p+1$, where the product $\left(s_{n-p+k} \cdots s_{n}\right)$ is omitted for $k=p+1$. The latter gives $c^{-1} c^{\prime} \in W_{n-p-1}$ by Proposition 12, so that $c^{\prime}=c d$ for some $d \in W_{n-p-1}$. Then $\bar{D}=\phi D$, where $D$ is the $i$-simplex of $\mathcal{C}^{n-p-1}$ with lift $d$.

We can now prove the proposition. Combining the first claim with the third in the case of 0 -simplices, we see that $\phi$ is an isomorphism between the vertex sets of $\mathcal{C}^{n-p-1}$ and $\operatorname{lk}_{\mathcal{C}^{n}}(C)$. The second and third claims then show that $\phi$ induces an isomorphism of simplicial complexes from $\mathcal{C}^{n-p-1}$ to $\operatorname{lk}_{\mathcal{C}^{n}}(C)$.

\section{The barycentric subdivision of $\mathcal{C}^{n}$ and the basic construction}

Our aim now is to complete the proof of Theorem 18 by proving Propositions 26 and 27 below. These results make use of the basic construction, whose definition we now recall from Section 2.5. Let $(W, S)$ be a Coxeter system. A mirrored space over $S$ is a space $X$ equipped with a mirror $X_{s} \subset X$ for each $s \in S$. Given such a mirrored space, the basic construction $\mathcal{U}(W, X)$ is then the quotient $(W \times X) / \sim$, where $(w, x) \sim(v, y)$ if and only if $x=y$ and $w^{-1} v$ lies in the subgroup generated by those $s \in S$ for which $x \in X_{s}$.

We will show in Proposition 26 that $\left|\operatorname{sd} \mathcal{C}^{n}\right|$ is the basic construction $\mathcal{U}\left(W_{n}, X\right)$ for an appropriate choice of mirrored space $X$. Then in Proposition 27 we will show that $\mathcal{U}\left(W_{n}, X\right)$ is $(n-1)$-connected. Together these show that the barycentric subdivision $\operatorname{sd} \mathcal{C}^{n}$ is $(n-1)$-connected, completing the proof of Theorem 18.

We begin by defining the required mirrored space $X$ over $S_{n}$. To do this we will identify a simplex $\Delta$ of $\operatorname{sd} \mathcal{C}^{n}$ and make its realization $|\Delta|$ into a mirrored space. 
Definition 21 (the simplex $\Delta$ ) For $i=0, \ldots, n$, let $a_{i}$ denote the $(n-i)$-simplex of $\mathcal{C}^{n}$ defined by

$$
a_{i}=\left\{\left(s_{i+1} \cdots s_{n}\right) W_{n-1}, \ldots, s_{n} W_{n-1}, e W_{n-1}\right\} .
$$

Each $a_{i}$ has lift $e \in W_{n}$. Now let $\Delta$ denote the $n$-simplex of $\operatorname{sd} \mathcal{C}^{n}$ defined by

$$
\Delta=\left\{a_{0}, \ldots, a_{n}\right\} .
$$

It is a simplex of $\operatorname{sd} \mathcal{C}^{n}$ since $a_{0} \supset \cdots \supset a_{n}$.

Definition 22 (the subcomplexes $\Delta_{s}$ ) For each $s \in S_{n}$, we define a subcomplex $\Delta_{s}$ of $\Delta$ as follows. If $s=s_{i}$ for $i=1, \ldots, n$, then $\Delta_{s_{i}}$ is the face

$$
\Delta_{s_{i}}=\left\{a_{0}, \ldots, \widehat{a_{i}}, \ldots, a_{n}\right\}
$$

of $\Delta$. If $s \in S_{0} \backslash S_{-1}$ then $\Delta_{s}$ is the face

$$
\Delta_{s}=\left\{a_{1}, \ldots, a_{n}\right\}
$$

of $\Delta$. And finally, if $s \in S_{-1}$ then

$$
\Delta_{s}=\Delta
$$

Definition 23 (the mirrored space $|\Delta|$ ) We make $|\Delta|$ into a mirrored space over $S_{n}$ by defining $|\Delta|_{s}=\left|\Delta_{s}\right| \subset|\Delta|$ for $s \in S_{n}$.

Example 24 ( $\Delta$ and $\Delta_{s}$ for Coxeter groups of type $A$ ) For the sequence $\left(A_{n}\right)_{n \geqslant 1}$, we saw in Example 2 that $W_{n}$ is the symmetric group $\Sigma_{n+1}$, and in Example 15 that $\mathcal{C}^{n}$ can be identified with the $n$-simplex $\Delta^{n}$. Under this identification the vertex $s_{i+1} \cdots s_{n} W_{n-1}$ is identified with $i$. Thus

$$
a_{i}=\left\{s_{i+1} \cdots s_{n} W_{n-1}, \ldots, s_{n} W_{n-1}, W_{n-1}\right\}=\{i+1, \ldots, n\} .
$$

Consequently $\Delta$ is the $n$-simplex of $\operatorname{sd} \Delta^{n}$ with vertices

$$
\{1, \ldots, n+1\}, \quad \ldots, \quad\{n, n+1\}, \quad\{n+1\} .
$$

This is illustrated in Figure 2 in the case $n=2$. Observe that in this case every 2 -simplex of sd $\Delta^{2}$ is a translate of $\Delta$ by an element of $\Sigma_{3}$, and that every simplex of $\operatorname{sd} \Delta^{2}$ is a face of such a translate.

The subcomplex $\Delta_{s_{i}}$ of $\Delta$ is simply the face opposite the vertex $a_{i}=\{i+1, \ldots, n\}$. This is illustrated in Figure 3 in the case $n=2$. Observe that $s_{1}$ fixes $\Delta_{s_{1}}$ vertexwise, and that $s_{2}$ fixes $\Delta_{s_{2}}$ vertexwise. 

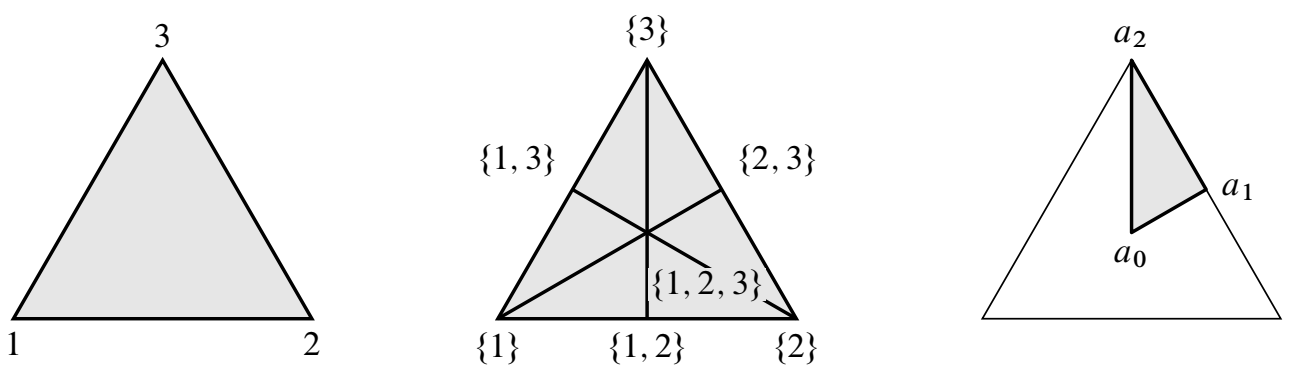

Figure 2: The simplex $\Delta^{2}$ (left), its subdivision $\mathrm{sd} \Delta^{2}$ (middle) and the simplex $\Delta$ (right)
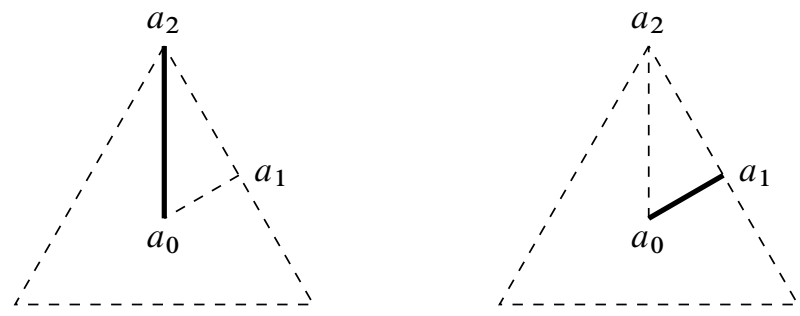

Figure 3: The faces $\Delta_{s_{1}}$ (left) and $\Delta_{s_{2}}$ (right)

The inclusion $|\Delta| \hookrightarrow\left|s d \mathcal{C}^{n}\right|$ extends uniquely to a $W_{n}$-equivariant map $W_{n} \times|\Delta| \rightarrow$ $\left|\operatorname{sd} \mathcal{C}^{n}\right|$. We want this to reduce to a map

$$
\mathcal{U}\left(W_{n},|\Delta|\right) \rightarrow\left|\operatorname{sd} \mathcal{C}^{n}\right|
$$

and so we must check that it respects the equivalence relation $\sim$ on $W_{n} \times|\Delta|$ that defines $\mathcal{U}\left(W_{n},|\Delta|\right)$. This is an immediate consequence of the following lemma.

Lemma 25 Under the action of $W_{n}$ on $\left|\operatorname{sd} \mathcal{C}^{n}\right|$, the mirror $|\Delta|_{s} \subset|\Delta| \subset\left|\operatorname{sd} \mathcal{C}^{n}\right|$ is fixed pointwise by $s$.

Proof Let $i \geqslant 0$. If $s \in S_{i} \backslash S_{i-1}$, then $s$ fixes $a_{j}$ for $j \neq i$. For $i \geqslant 1$ this follows from Proposition 8, and for $i=0$ it follows because $s$ commutes with $s_{k}$ for $k \geqslant 2$. Similarly, if $s \in S_{-1}$ then $s$ fixes $a_{j}$ for all $j$. In all cases it follows that $s$ fixes every vertex of $\Delta_{s}$, so that $s$ fixes $|\Delta|_{s}=\left|\Delta_{s}\right|$ pointwise.

We can now state the main results of this section.

Proposition 26 The $\operatorname{map} \mathcal{U}\left(W_{n},|\Delta|\right) \rightarrow\left|\operatorname{sd} \mathcal{C}^{n}\right|$ is a homeomorphism.

Proposition $27 \mathcal{U}\left(W_{n},|\Delta|\right)$ is $(n-1)$-connected. 
Example 28 (the map $\mathcal{U}\left(W_{1},|\Delta|\right) \rightarrow\left|\operatorname{sd} \mathcal{C}^{1}\right|$ for groups of type $B$ ) Let us illustrate Proposition 26 in the case of the sequence $\left(B_{n+1}\right)_{n \geqslant 1}$ and $n=1$. As in Example 3, the Coxeter diagram of $W_{1}$ is as follows:

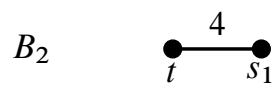

In this case $\Delta$ is the simplex with vertex set $\left\{a_{0}, a_{1}\right\}$, while $\Delta_{s_{1}}$ and $\Delta_{t}$ are the faces with vertices $a_{0}$ and $a_{1}$ respectively. Thus $|\Delta|$ is an interval and $|\Delta|_{s_{1}}$ and $\left|\Delta_{t}\right|$ are its endpoints. We draw $|\Delta|$ as follows, with $|\Delta|_{s_{1}}$ represented by a hollow vertex and $|\Delta|_{t}$ represented by a solid vertex:

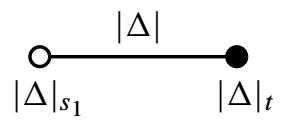

Then by definition $\mathcal{U}\left(W_{1},|\Delta|\right)$ is the union of the translates of $|\Delta|$ by elements of $W_{1}$, where for each $w \in W_{1}$, the solid vertices of $w|\Delta|$ and $w t|\Delta|$ are identified, as are the hollow vertices of $w|\Delta|$ and $w s_{1}|\Delta|$. Thus $\mathcal{U}\left(W_{1},|\Delta|\right)$ is as shown on the left of Figure 4.
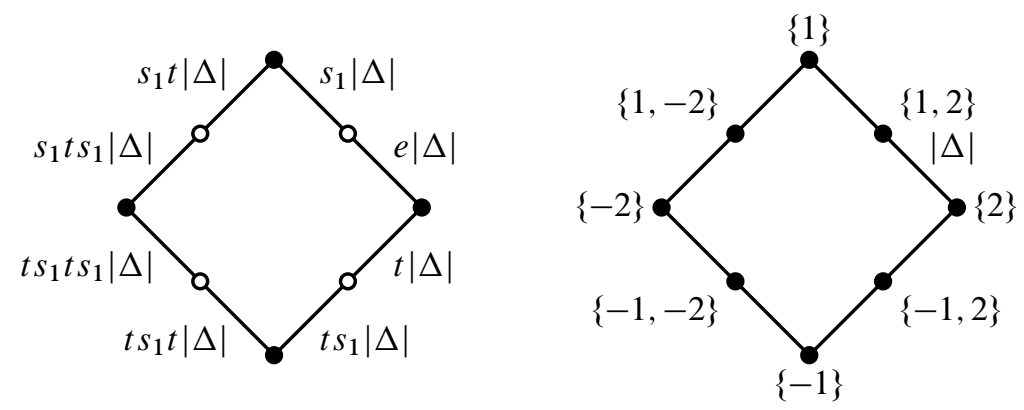

Figure 4: The spaces $\mathcal{U}\left(W_{1},|\Delta|\right)$ and $\left|\operatorname{sd} \mathcal{C}^{1}\right|$

Recall from Example 16 that $\mathcal{C}^{1}$ is the square with vertices $\{ \pm 1, \pm 2\}$, with each vertex being opposite to its negative, where $t$ and $s_{1}$ act as the permutations $(1,-1)$ and $(1,2)(-1,-2)$ respectively. Thus $\left|\operatorname{sd} \mathcal{C}^{1}\right|$ is as shown on the right of Figure 4 with the subspace $|\Delta|$ labelled. Now the map $\mathcal{U}\left(W_{1},|\Delta|\right) \rightarrow\left|\operatorname{sd}^{1}\right|$ is the one that is evident from the drawings. It is the identity on the copy of $|\Delta|$ within each space, it is equivariant with respect to the $W_{1}$-actions, and it is clearly a homeomorphism.

We now work towards the proof of Proposition 26. It relies on the following two lemmas. Roughly speaking, these correspond to surjectivity and injectivity of the map $\mathcal{U}\left(W_{n},|\Delta|\right) \rightarrow\left|\operatorname{sd} \mathcal{C}^{n}\right|$ respectively. 
Lemma 29 Every $n$-simplex of $\operatorname{sd} \mathcal{C}^{n}$ is a translate of $\Delta$, and every simplex of $\operatorname{sd} \mathcal{C}^{n}$ is a face of such a translate.

Proof Let $\boldsymbol{C}=\left\{C_{0}, \ldots, C_{n}\right\}$ be an $n$-simplex of sd $\mathcal{C}^{n}$ with $C_{0} \subset \cdots \subset C_{n}$. Then $\boldsymbol{C}$ induces a natural ordering of the vertices of $C_{n}$ by declaring that each $C_{i}$ consists of an initial segment. Let $c$ be a lift of $C_{n}$ that induces this ordering. Then, by inspecting the definition of the induced order, one sees that $C=c \cdot \Delta$ as required. This proves the first part. Now observe that every simplex of $\mathcal{C}^{n}$ is a face of an $n$-simplex, since a simplex with a given lift is a face of the $n$-simplex with that lift. The second part follows.

Lemma 30 Let $F$ be a face of $\Delta$. Then the stabilizer of $F$ under the action of $W_{n}$ is the subgroup generated by those $s \in S_{n}$ for which $F \subset \Delta_{s}$.

Proof The stabilizer of a simplex of $\operatorname{sd}^{n}$ coincides with the intersection of the stabilizers of its vertices. To see this, let $w \in W_{n}$ and let $C=\left\{C_{0}, \ldots, C_{k}\right\}$ be a simplex of $\operatorname{sd} \mathcal{C}^{n}$ that is fixed by $w$. Then each $C_{i}$ is a simplex of $\mathcal{C}^{n}$ itself, and without loss $C_{0} \subset \cdots \subset C_{k}$. The assumption $w \cdot C=C$ means that $w$ permutes the $C_{i}$. But since each $C_{i}$ has a different cardinality, this means that $w$ must in fact fix each $C_{i}$. So the stabilizer of $\boldsymbol{C}$ is contained in the intersection of the stabilizers of its vertices. The converse is immediate. See [4, page 115].

For the purposes of this proof, given $i \geqslant 0$ we write $S_{=i}$ for the difference $S_{i} \backslash S_{i-1}$. So for $i \geqslant 1$ we have $S_{=i}=\left\{s_{i}\right\}$, while $S_{=0}$ is the set of elements of $S_{0}$ that do not commute with $s_{1}$.

Fix $i \geqslant 0$, and consider the vertex $a_{i}$ of $\operatorname{sd} \mathcal{C}^{n}$. We will show that the stabilizer of $a_{i}$ is the subgroup of $W_{n}$ generated by

$$
S_{n} \backslash S_{=i}=S_{i-1} \cup\left\{s_{i+1}, \ldots, s_{n}\right\} .
$$

To see this, recall that the vertices of $a_{i}$ (when $a_{i}$ is regarded as a simplex of $\mathcal{C}^{n}$ ) are

$$
s_{i+1} \cdots s_{n} W_{n-1}, \ldots, s_{n} W_{n-1}, W_{n-1} .
$$

Observe that if $s \in S_{i-1}$ then $s$ commutes with $s_{i+1}, \ldots, s_{n}$, and so fixes the vertices of $a_{i}$, and so fixes $a_{i}$ itself. And if $s \in\left\{s_{i+1}, \ldots, s_{n}\right\}$, then by Proposition $8, s$ permutes the vertices of $a_{i}$, and so fixes $a_{i}$ itself. So the subgroup generated by $S_{n} \backslash S_{=i}$ fixes $a_{i}$. Conversely, suppose that $w \in W_{n}$ fixes $a_{i}$. Proposition 8 shows that any permutation of the vertices of $a_{i}$ can be achieved using the subgroup generated by $s_{i+1}, \ldots, s_{n}$. So after left-multiplying $w$ by an element of the subgroup generated by $s_{i+1}, \ldots, s_{n}$, we may assume that $w$ fixes every vertex of $a_{i}$. Proposition 12 now shows that $w$ lies in the subgroup generated by $S_{i-1}$, as required. 
Let $F=\left\{a_{i_{1}}, \ldots, a_{i_{r}}\right\}$. Then according to the first paragraph, the stabilizer of $F$ is the intersection of the stabilizers of the $a_{i_{j}}$. By the last paragraph this is the intersection of the subgroups generated by the sets $S_{n} \backslash S_{=i_{j}}$, and by the general results described in Section 2.4, this is the subgroup generated by $\bigcap\left(S_{n} \backslash S_{=i_{j}}\right)=S_{n} \backslash \bigcup S_{=i_{j}}$.

It remains to show that $S_{n} \backslash \bigcup S_{=i_{j}}$ is the set of $s$ such that $F \subset \Delta_{s}$. Now by the definition of $\Delta_{s}$, we see that $F \subset \Delta_{s}$ for all $s \in S_{-1}$, and that $F \subset \Delta_{s}$ for $s \in S_{=i}$ if and only if $a_{i} \notin F$. Thus the set of $s$ such that $F \subset \Delta_{s}$ is $S \backslash \bigcup S_{=i_{j}}$ as required.

Example 31 Let us illustrate the proof of Lemma 30 for the sequence $\left(A_{n}\right)_{n \geqslant 1}$ and $n=2$. We described $\mathcal{C}^{2}, \operatorname{sd} \mathcal{C}^{2}, \Delta$ and the $\Delta_{s}$ for this case in Example 24. The following points correspond to the paragraphs of the proof.

- First observe that the action of $W_{2}=\Sigma_{3}$ on the vertices of $\mathrm{sd} \mathcal{C}^{2}$ has three orbits, namely the three vertices of the triangle (which are the 0 -simplices of $\mathcal{C}^{2}$ ), the midpoints of the edges of the triangle (which are the 1 -simplices of $\mathcal{C}^{2}$ ), and the barycentre of the triangle (which is the single 2-simplex of $\mathcal{C}^{2}$ ). Each simplex of $\operatorname{sd} \mathcal{C}^{2}$ contains at most one vertex from each orbit. So the stabilizer of a simplex is the intersection of the stabilizers of its orbits.

- We have $S_{2}=\left\{s_{1}, s_{2}\right\}, S_{1}=\left\{s_{1}\right\}$ and $S_{0}=\varnothing$. Thus $S_{=2}=\left\{s_{2}\right\}, S_{=1}=\left\{s_{1}\right\}$ and $S_{=0}=\varnothing$.

- Next, observe that the stabilizers of $a_{0}, a_{1}$ and $a_{2}$ are $\left\langle s_{1}, s_{2}\right\rangle,\left\langle s_{2}\right\rangle$ and $\left\langle s_{1}\right\rangle$ respectively, and these are indeed the subgroups generated by the sets $S_{2} \backslash S_{=0}$, $S_{2} \backslash S_{=1}$ and $S_{2} \backslash S_{=2}$ respectively. So $s_{i}$ stabilizes $a_{j}$ if and only if $i \neq j$.

- By the first point, the stabilizer of a face $F$ of $\Delta$ is the intersection of the stabilizers of its vertices, and by the previous point this is the subgroup generated by the $s_{i}$ for which $a_{i}$ is not contained in $F$.

- On the other hand, $F \subset \Delta_{s_{i}}$ if and only if $F$ does not contain $a_{i}$. This, combined with the previous point, shows that the stabilizer of $F$ is generated by the $s$ for which $F \subset \Delta_{S}$.

Proof of Proposition 26 The map is surjective because any point of $\left|\mathrm{sd} \mathcal{C}^{n}\right|$ is in a translate of $|\Delta|$. This follows from Lemma 29, which shows that every simplex of $\operatorname{sd} \mathcal{C}^{n}$ is a face of a translate of $\Delta$.

To show that the map is injective, suppose that $[w, x],[v, y] \in \mathcal{U}\left(W_{n},|\Delta|\right)$ have the same image in $\left|\operatorname{sd} \mathcal{C}^{n}\right|$, or in other words that $w \cdot x=v \cdot y$. We will show that $(w, x) \sim(v, y)$ so that $[w, x]=[v, y]$. First we show that $x=y$. There is a canonical map $\operatorname{sd} \mathcal{C}^{n} \rightarrow \Delta^{n}$ that sends a vertex $C$ of $\operatorname{sd} \mathcal{C}^{n}$, or in other words a simplex $C$ of $\mathcal{C}^{n}$, to the vertex $|C|$ of $\Delta^{n}$. By construction this is $W_{n}$-invariant and restricts 
to an isomorphism $\Delta \rightarrow \Delta^{n}$. Taking realizations, we obtain a $W_{n}$-invariant map $\left|\operatorname{sd} \mathcal{C}^{n}\right| \rightarrow \Delta^{n}$ that restricts to a homeomorphism $|\Delta| \rightarrow \Delta^{n}$. Since $w \cdot x=v \cdot y$, we therefore have $x=y$. Next we show that $w^{-1} v$ lies in the subgroup generated by those $s \in S_{n}$ for which $x \in|\Delta|_{s}$. Write $F$ for the unique face of $\Delta$ for which $x$ lies in the interior of $F$. Then $x \in|\Delta|_{s}=\left|\Delta_{s}\right|$ if and only if $F \subset \Delta_{s}$. Moreover, since $w \cdot x=v \cdot y$ and $x=y$, we see that $w^{-1} v$ lies in the stabilizer of $x$, which is exactly the stabilizer of $F$. Then $w^{-1} v$ lies in the claimed subgroup by Lemma 30 . Consequently $(w, x) \sim(v, y)$ as required.

The map is a homeomorphism because $\left|s d \mathcal{C}^{n}\right|$ has the weak topology with respect to the realizations of its simplices. By Lemma 29 this coincides with the weak topology with respect to the realizations of its $n$-simplices. This is exactly the topology of $\mathcal{U}\left(W_{n},|\Delta|\right)$.

We now work towards the proof of Proposition 27. We will make use of the increasing union of chambers, which we described in Section 2.6. Recall in particular that if $w \in W_{n}$ then $\operatorname{In}(w)=\{s \in S \mid \ell(w s)<\ell(w)\}$ is the set of letters with which a reduced expression for $w$ can end. We begin with two lemmas.

Lemma 32 For $w \in W_{n}, w \neq e$, the space $|\Delta|^{\operatorname{In}(w)}$ is $(n-2)$-connected.

Proof The set $\operatorname{In}(w)$ is nonempty since $w \neq e$. Thus $|\Delta|^{\operatorname{In}(w)}$ is either $|\Delta|$, or it is a nonempty union of facets of $|\Delta|$. In the first case it is contractible, and in the second case it is either contractible (if not all facets are in the union) or it is $\partial|\Delta| \cong S^{n-1}$ (if all facets are in the union). In all cases it is $(n-2)$-connected.

Lemma 33 Let $n \geqslant 1$. Suppose that $(X ; A, B)$ is a $C W$-triad in which $A$ and $B$ are $(n-1)$-connected and $C=A \cap B$ is (n-2)-connected. Then $X$ is (n-1)-connected.

Proof For $n=1$ this is immediate since the union of two path-connected spaces with nonempty intersection is path-connected. So we assume that $n \geqslant 2$. The pairs $(A, C)$ and $(B, C)$ are $(n-1)$-connected, and $C$ is path-connected, so Theorem 4.23 of [13] can be applied to show that $\pi_{i}(A, C) \rightarrow \pi_{i}(X, B)$ is an isomorphism for $i<2 n-2$, and in particular for $i \leqslant(n-1)$. Thus $(X, B)$ is $(n-1)$-connected, and the same then follows for $X$ itself.

Proof of Proposition 27 If $n=0$ then the claim is that $\mathcal{U}\left(W_{n},|\Delta|\right)$ is nonempty, which holds vacuously. So we may assume that $n \geqslant 1$. 
As in Section 2.6, order the elements of $W_{n}$ as $w_{0}, w_{1}, w_{2}, \ldots$ starting with the identity and respecting the length. Then $\mathcal{U}\left(W_{n},|\Delta|\right)$ is the union of subcomplexes $P_{0} \subset P_{1} \subset P_{2} \subset \cdots$, where $P_{0}=|\Delta|$ and

$$
P_{m}=P_{m-1} \cup w_{m}|\Delta| \text { with } \quad P_{m-1} \cap w_{m}|\Delta|=w_{m}|\Delta|^{\operatorname{In}\left(w_{m}\right)} .
$$

It will suffice to show that each $P_{m}$ is $(n-1)$-connected. We do this by induction on $m$.

In the initial case $m=0$ we have $P_{0}=e|\Delta|$, which is contractible and so the claim holds. For the induction step we take $m \geqslant 1$ and assume that $P_{m-1}$ is $(n-1)-$ connected. Then $P_{m}=P_{m-1} \cup w_{m}|\Delta|$ is the union of the subcomplexes $P_{m-1}$ and $w_{m}|\Delta|$, and their intersection $w_{m}|\Delta|^{\operatorname{In}\left(w_{m}\right)}$ is $(n-2)$-connected by Lemma 32. Thus $\left(P_{m} ; P_{m-1}, w_{m}|\Delta|\right)$ is a CW-triad in which the subspaces $P_{m-1}$ and $w_{m}|\Delta|$ are $(n-1)$-connected and their intersection is $(n-2)-$ connected. It now follows from Lemma 33 that $P_{m}$ is $(n-1)-$ connected as required.

\section{The semisimplicial set $\mathcal{D}^{n}$}

In this section we introduce a semisimplicial set $\mathcal{D}^{n}$ with an action of $W_{n}$. It will be used in the next section to give the proof of the Main Theorem. As explained in Section 1.5, the definition of $\mathcal{D}^{n}$ is inspired by the "complex of injective words" (see Example 35) which is used in existing proofs of homological stability for symmetric groups, for example $[21 ; 19 ; 25]$. Indeed, we first obtained $\mathcal{D}^{n}$ by writing every aspect of the complex of injective words in terms of the symmetric groups and adjacent transpositions, and then abstracting this definition to our sequences of Coxeter groups $\left(W_{n}\right)_{n \geqslant 1}$. This may leave the definition of $\mathcal{D}^{n}$ a little unmotivated, but we hope that it will become apparent over this section and the next that $\mathcal{D}^{n}$ is precisely the object required to complete the proof of the main theorem.

The main result of the section is that the realization $\left\|\mathcal{D}^{n}\right\|$ is $(n-1)$-connected. This is proved by identifying $\mathcal{D}^{n}$ as the semisimplicial set of ordered simplices in $\mathcal{C}^{n}$, and then using the fact that $\mathcal{C}^{n}$ is weakly Cohen-Macaulay of dimension $n$ to deduce that the geometric realization $\left\|\mathcal{D}^{n}\right\|$ is $(n-1)$-connected. We learned this approach from Wahl's paper[28], in particular Proposition 7.9, which is due to Randal-Williams.

In this section and the next we will use semisimplicial spaces and their realizations. The background material we require can be found in Section 2 of [25].

Definition 34 Let $\mathcal{D}^{n}$ denote the semisimplicial set with $k$-simplices

$$
\mathcal{D}_{k}^{n}= \begin{cases}W_{n} / W_{n-k-1} & \text { for } k \leqslant n, \\ \varnothing & \text { for } k>n,\end{cases}
$$


and with face maps

$$
d_{i}: W_{n} / W_{n-k-1} \rightarrow W_{n} / W_{n-k}
$$

defined by

$$
d_{i}\left(c W_{n-k-1}\right)=c\left(s_{n-k+i} \cdots s_{n-k+1}\right) W_{n-k}
$$

for $i=0, \ldots, k$. Here the product $\left(s_{n-k+i} \cdots s_{n-k+1}\right)$ is omitted when $i=0$.

One can verify directly that the face maps $d_{i}$ satisfy the relations $d_{i} \circ d_{j}=d_{j-1} \circ d_{i}$ for $i<j$. Alternatively, it is a consequence of the proof of Proposition 37 below.

Example $35\left(\mathcal{D}^{n}\right.$ for groups of type $A, B$ and $\left.D\right)$ In order to illustrate the definition above, we recall the definition of the complex of injective words. Let $L$ be a set. An injective word in $L$ is a finite sequence of distinct elements of $L$. The complex of injective words in $L$ is the semisimplicial set whose $k$-simplices are injective words in $L$ of length $(k+1)$, and in which the face map $d_{i}$ sends $\left(x_{0}, \ldots, x_{k}\right)$ to $\left(x_{0}, \ldots, \widehat{x_{i}}, \ldots, x_{k}\right)$.

For the family $\left(A_{n}\right)_{n \geqslant 1}$, the semisimplicial set $\mathcal{D}^{n}$ is the complex of injective words in $\{1, \ldots, n+1\}$. Recall from Example 2 that $W_{n}$ is the group of permutations of $\{1, \ldots, n+1\}$. Thus $W_{n} / W_{n-k-1}$ can be identified with the set of injective words of length $(k+1)$ in $\{1, \ldots, n+1\}$ via the isomorphism

$$
\sigma W_{n-k-1} \longmapsto(\sigma(n-k+1), \ldots, \sigma(n+1)) \text {. }
$$

To see that the face map $d_{i}$ corresponds to the map that deletes the $i^{\text {th }}$ letter, we must show that

$$
\begin{aligned}
\left(\sigma s_{n-k+i} \cdots s_{n-k+1}(n-k+2),\right. & \left.\ldots, \sigma s_{n-k+i} \cdots s_{n-k+1}(n+1)\right) \\
& =(\sigma(n-k+1), \ldots, \widehat{\sigma(n-k+i)}, \ldots, \sigma(n+1)) .
\end{aligned}
$$

This follows because $s_{n-k+i} \cdots s_{n-k+1}$ decreases each of $(n-k+2), \ldots,(n-k+i)$ by one, sends $(n-k+i)$ to $(n-k+1)$, and fixes $(n-k+i+1), \ldots,(n+1)$.

For the family $\left(B_{n+1}\right)_{n \geqslant 1}, \mathcal{D}^{n}$ is the subset of the complex of injective words in $\{ \pm 1, \ldots, \pm(n+1)\}$ in which each word features at most one entry from each pair $\{i,-i\}$. For the family $\left(D_{n+2}\right)_{n \geqslant 1}$, we have that $\mathcal{D}^{n}$ is the subset of the complex of injective words in $\{ \pm 1, \ldots, \pm(n+2)\}$ in which each word again features at most one entry from each pair $\{i,-i\}$. These two facts can be proved by the method of the previous example, this time making use of Examples 3 and 4. 
Definition 36 Let $X$ be a simplicial complex. By an ordered simplex of $X$, we mean a simplex of $X$ equipped with an ordering of its vertices. The semisimplicial set of ordered simplices in $X$, denoted $X^{\text {ord }}$, has for its $k$-simplices the ordered $k$-simplices in $X$, with face maps $d_{i}$ given by forgetting the $i^{\text {th }}$ vertex of an ordered simplex.

Proposition $37 \mathcal{D}^{n}$ is isomorphic to $\mathcal{C}^{n \text {,ord }}$.

Proof We define $\phi_{k}: \mathcal{D}_{k}^{n} \rightarrow \mathcal{C}_{k}^{n \text {,ord }}$ by

$$
\phi_{k}\left(c W_{n-k-1}\right)=\left\{c\left(s_{n-k+1} \cdots s_{n}\right) W_{n-1}, \ldots, c s_{n} W_{n-1}, c W_{n-1}\right\}
$$

for $c W_{n-k-1} \in W_{n} / W_{n-k-1}$. In other words, $\phi_{k}\left(c W_{n-k-1}\right)$ is the $k$-simplex with lift $c$, equipped with the ordering induced by $c$. The map $\phi_{k}$ is well defined because the generators of $W_{n-k-1}$ all commute with $s_{n-k+1}, \ldots, s_{n}$. It is surjective because by definition every simplex admits a lift, and any ordering of a simplex is afforded by some lift (see the paragraph following Definition 13). It is injective because if $\phi_{k}\left(c W_{n-k-1}\right)=\phi_{k}\left(c^{\prime} W_{n-k-1}\right)$ then $c s_{i} \cdots s_{n} W_{n-1}=c^{\prime} s_{i} \cdots s_{n} W_{n-1}$ for $i=n-k+1, \ldots, n+1$, so that $c W_{n-k-1}=c^{\prime} W_{n-k-1}$ by Proposition 12 .

To complete the proof we must show that the face maps in $\mathcal{C}^{n \text {,ord }}$ and $\mathcal{D}^{n}$ are compatible under the $\phi_{k}$. In other words, given $0 \leqslant i \leqslant k \leqslant n$, we must show that

$$
\phi_{k-1} \circ d_{i}=d_{i} \circ \phi_{k} \text {. }
$$

Observe from the definition of $d_{i}$ in $\mathcal{D}^{n}$ that $d_{i}\left(c W_{n-k-1}\right)=d_{i-1}\left(c s_{n-k+i} W_{n-k+1}\right)$ for $i \geqslant 1$. Proposition 8 shows that $\phi_{k}\left(c W_{n-k-1}\right)$ and $\phi_{k}\left(c s_{n-k+i} W_{n-k-1}\right)$ differ only by the transposition of their $(i-1)^{\mathrm{st}}$ and $i^{\text {th }}$ vertices, so that $d_{i}\left(\phi_{k}\left(c W_{n-k-1}\right)\right)=$ $d_{i-1}\left(\phi_{k}\left(c s_{n-k+i} W_{n-k-1}\right)\right)$. Thus the claim will follow by induction on $i$ so long as we can show that

$$
\phi_{k-1} \circ d_{0}=d_{0} \circ \phi_{k}
$$

This follows by inspection.

Corollary $38\left\|\mathcal{D}^{n}\right\|$ is $(n-1)$-connected.

Proof Theorem 18 shows that $\mathcal{C}^{n}$ is weakly Cohen-Macaulay of dimension $n$. It was shown in Proposition 7.9 of [28] that if a simplicial complex $X$ is weakly CohenMacaulay of dimension $n$, then $\left\|X^{\text {ord }}\right\|$ is $(n-1)$-connected. Consequently $\left\|\mathcal{C}^{n, \text { ord }}\right\|$ is $(n-1)$-connected, and by Proposition 37 the same holds for $\left\|\mathcal{D}^{n}\right\|$.

Remark 39 In the case of the sequence $\left(A_{n}\right)_{n \geqslant 1}$, when $\mathcal{D}^{n}$ is the complex of injective words in $\{1, \ldots, n+1\}$, the connectivity of $\left\|\mathcal{D}^{n}\right\|$ is well-known: see $[9 ; 2 ; 19 ; 25]$. (Strictly speaking, the first and third references deal with the homology of $\left\|\mathcal{D}^{n}\right\|$, rather than its homotopy type.) 


\section{Completion of the proof}

We now complete the proof of the Main Theorem. This section is modelled closely on Section 5 of [25], from which there is little essential difference. It is also similar to the proof of Theorem 2 of [19].

We regard $\mathcal{D}^{n}$ as a simplicial space by equipping its constituent sets with the discrete topology. Then we form a semisimplicial space

$$
E W_{n} \times_{W_{n}} \mathcal{D}^{n}
$$

by setting $\left(E W_{n} \times W_{n} \mathcal{D}^{n}\right)_{k}=E W_{n} \times{ }_{W_{n}} \mathcal{D}_{k}^{n}$ and using the face maps obtained from those of $\mathcal{D}^{n}$.

Lemma 40 The projection $E W_{n} \times_{W_{n}} \mathcal{D}_{0}^{n} \rightarrow B W_{n}$ makes $E W_{n} \times_{W_{n}} \mathcal{D}^{n}$ into an augmented simplicial space over $B W_{n}$, and the induced map $\left\|E W_{n} \times{ }_{W_{n}} \mathcal{D}^{n}\right\| \rightarrow B W_{n}$ is $(n-1)$-connected.

Proof The composites of the projection with $d_{0}$ and $d_{1}$ coincide, so that the projection is indeed an augmentation. Since the map $E W_{n} \rightarrow B W_{n}$ is a locally trivial principal $W_{n}$-bundle, it follows that $\left\|E W_{n} \times{ }_{W_{n}} \mathcal{D}^{n}\right\| \rightarrow B W_{n}$ is a locally trivial bundle with fibre $\left\|W_{n} \times W_{n} \mathcal{D}^{n}\right\| \cong\left\|\mathcal{D}^{n}\right\|$, which is $(n-1)$-connected by Corollary 38 , so that the map itself is $(n-1)$-connected.

Lemma 41 There are homotopy equivalences $E W_{n} \times{ }_{W_{n}} \mathcal{D}_{k}^{n} \simeq B W_{n-k-1}$ under which the face maps $d_{i}: E W_{n} \times_{W_{n}} \mathcal{D}_{k}^{n} \rightarrow E W_{n} \times W_{n} \mathcal{D}_{k-1}^{n}$ are all homotopic to the stabilization map $B W_{n-k-1} \rightarrow B W_{n-k}$, and under which the composite

$$
E W_{n} \times_{W_{n}} \mathcal{D}_{0}^{n} \rightarrow\left\|E W_{n} \times_{W_{n}} \mathcal{D}^{n}\right\| \rightarrow B W_{n}
$$

is homotopic to the stabilization map $B W_{n-1} \rightarrow B W_{n}$.

Proof There is an isomorphism

$$
E W_{n} \times_{W_{n}} \mathcal{D}_{k}^{n}=E W_{n} \times_{W_{n}}\left(W_{n} / W_{n-k-1}\right) \stackrel{\cong}{\longrightarrow} E W_{n} / W_{n-k-1}
$$

sending the orbit of $\left(x, c W_{n-k-1}\right)$ to the orbit of $c^{-1} x$. This identifies $d_{i}$ with the map

$$
E W_{n} / W_{n-k-1} \rightarrow E W_{n} / W_{n-k}
$$

sending the $W_{n-k-1}$-orbit of $x$ to the $W_{n-k}$-orbit of $\left(s_{n-k+1} \cdots s_{n-k+i}\right) x$. We claim that this map is homotopic to the one sending the $W_{n-k-1}$-orbit of $x$ to the $W_{n-k}$-orbit of $x$. Indeed, $E W_{n}$ is contractible, and $W_{n-k-1}$ acts on it freely. Moreover, when we 
equip $E W_{n}$ with its natural $\mathrm{CW}$-structure as the realization of a simplicial set, then this action is cellular. It follows that any two $W_{n-k-1}$-equivariant maps from $E W_{n}$ to itself are $W_{n-k-1}$-equivariantly homotopic. (This can be proved by induction on the cells. Alternatively, see [20, Definition 1.8 and Theorem 1.9] in the case where $G=W_{n-k-1}$ and $\mathcal{F}$ consists of the trivial subgroup.) Since $\left(s_{n-k+1} \cdots s_{n-k+i}\right)$ commutes with every element of $W_{n-k-1}$, the map $E W_{n} \rightarrow E W_{n}$ given by left-multiplication by $\left(s_{n-k+1} \cdots s_{n-k+i}\right)$ is $W_{n-k-1}$-equivariant, and is therefore $W_{n-k-1}$-equivariantly homotopic to the identity map. The claim now follows by taking $W_{n-k-1}$-orbits in the domain and $W_{n-k}$-orbits in the codomain.

Now the equivariant homotopy equivalences

$$
E W_{n-k-1} \rightarrow E W_{n} \quad \text { and } \quad E W_{n-k} \rightarrow E W_{n}
$$

induce homotopy equivalences

$$
B W_{n-k-1} \rightarrow E W_{n} / W_{n-k-1} \quad \text { and } \quad B W_{n-k} \rightarrow E W_{n} / W_{n-k}
$$

under which the map $E W_{n} / W_{n-k-1} \rightarrow E W_{n} / W_{n-k}$ just described becomes the stabilization map.

The skeletal filtration of $\left\|E W_{n} \times_{W_{n}} \mathcal{D}^{n}\right\|$ leads to a first-quadrant spectral sequence

$$
E_{k, l}^{1}=H_{l}\left(E W_{n} \times_{W_{n}} \mathcal{D}_{k}^{n}\right) \Longrightarrow H_{k+l}\left(\left\|E W_{n} \times_{W_{n}} D^{n}\right\|\right)
$$

in which the differential $d^{1}$ is given by the alternating sum $\sum_{i=0}^{k}(-1)^{i}\left(d_{i}\right)_{*}$ of the maps induced by the face maps. Lemma 41 allows us to identify the $E^{1}$-term of this spectral sequence: $E_{k, l}^{1}=H_{l}\left(B W_{n-k-1}\right)$, and $d^{1}: E_{k, l}^{1} \rightarrow E_{k-1, l}^{1}$ is the map $H_{l}\left(B W_{n-k-1}\right) \rightarrow H_{l}\left(B W_{n-k}\right)$ induced by stabilization if $k$ is even, and is zero if $k$ is odd.

Lemma 42 For all $m<n$, assume that the stabilization map $H_{l}\left(B W_{m-1}\right) \rightarrow H_{l}\left(B W_{m}\right)$ is an isomorphism in degrees $2 l \leqslant m$. Then the spectral sequence has the following properties:

(1) $E_{0, l}^{\infty}=\cdots=E_{0, l}^{2}=E_{0, l}^{1}$ for $2 l \leqslant n$.

(2) $E_{k, l}^{\infty}=0$ for $k>0$ and $2(k+l) \leqslant n$.

Proof The assumption allows us to deduce that $E_{k, l}^{2}=0$ when $k \geqslant 1$ is odd and $2 l+k+1 \leqslant n$, and that $E_{k, l}^{2}=0$ when $k \geqslant 2$ is even and $2 l+k \leqslant n$. For in the first case

$$
d^{1}: E_{k+1, l}^{1} \rightarrow E_{k, l}^{1}
$$


is the stabilization map

$$
H_{l}\left(B W_{n-k-2}\right) \rightarrow H_{l}\left(B W_{n-k-1}\right),
$$

and in the second case

$$
d^{1}: E_{k, l}^{1} \rightarrow E_{k-1, l}^{1}
$$

is the stabilization map

$$
H_{l}\left(B W_{n-k-1}\right) \rightarrow H_{l}\left(B W_{n-k}\right),
$$

and our assumption means that both are isomorphisms in the given range. Figure 5 shows the $E^{1}$-page, where the left-hand shaded region consists of terms with total degree satisfying $2 * \leqslant n$ and the right-hand shaded region consists of terms that vanish on the $E^{2}$-page.

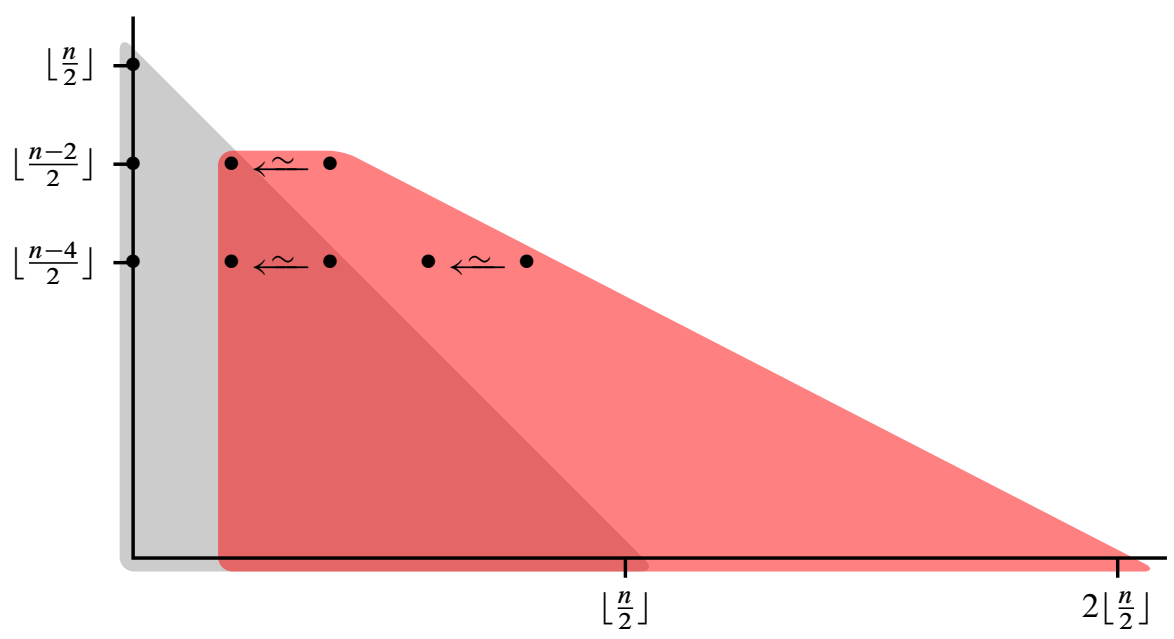

Figure 5: The spectral sequence $\left(E_{s, t}^{r}\right)$

To prove the first claim, observe that, since $d^{1}: E_{1, l}^{1} \rightarrow E_{0, l}^{1}$ is zero, $E_{0, l}^{2}=E_{0, l}^{1}$. The remaining differentials with target in bidegree $(0, l)$ are

$$
d^{k}: E_{k, l-k+1}^{k} \rightarrow E_{0, l}^{k}
$$

with $k \geqslant 2$, and these have domain zero since

$$
2(l-k+1)+k \leqslant 2(l-k+1)+k+1=2 l-k+2 \leqslant 2 l \leqslant n
$$

so that $E_{k, l-k+1}^{2}=0$. To prove the second claim, observe that if $2(k+l) \leqslant n$ and $k>0$, then certainly

$$
2 l+k<2 l+k+1 \leqslant 2(l+k) \leqslant n,
$$


so that $E_{k, l}^{2}=0$.

We can now complete the proof of the main theorem, showing by induction on $n \geqslant 0$ that $H_{l}\left(B W_{n-1}\right) \rightarrow H_{l}\left(B W_{n}\right)$ is an isomorphism for $2 l \leqslant n$. (In the main theorem this claim was made only for $n \geqslant 2$, but the proof by induction relies on the cases obtained by extending to the left.) For $n=0$ the claim is that $H_{0}\left(B W_{-1}\right) \rightarrow H_{0}\left(B W_{0}\right)$ is an isomorphism, which is trivial since $B W_{-1}$ and $B W_{0}$ are both path connected. Take $n \geqslant 1$ and suppose that the theorem holds for all smaller integers. Lemma 41 shows that the composite

$$
H_{l}\left(B W_{n-1}\right)=E_{0, l}^{1} \rightarrow E_{0, l}^{\infty} \rightarrow H_{l}\left(\left\|E W_{n} \times_{W_{n}} \mathcal{D}^{n}\right\|\right) \rightarrow H_{l}\left(B W_{n}\right),
$$

is the stabilization map, and we must show that this is an isomorphism for $2 l \leqslant n$. Lemma 42 shows that the first two arrows are isomorphisms in this range, while Lemma 40 shows that the last map is an isomorphism for $l \leqslant n-1$, which holds since $2 l \leqslant n$ and $n \geqslant 2$.

\section{References}

[1] A Björner, F Brenti, Combinatorics of Coxeter groups, Graduate Texts in Mathematics 231, Springer, New York (2005) MR2133266

[2] A Björner, M Wachs, On lexicographically shellable posets, Trans. Amer. Math. Soc. 277 (1983) 323-341 MR690055

[3] N Bourbaki, Lie groups and Lie algebras, Chapters 4-6, Elements of Mathematics, Springer, Berlin (2002) MR1890629

[4] GE Bredon, Introduction to compact transformation groups, Pure and Applied Mathematics 46, Academic Press, New York (1972) MR0413144

[5] M R Bridson, A Haefliger, Metric spaces of non-positive curvature, Grundl. Math. Wissen. 319, Springer, Berlin (1999) MR1744486

[6] D Calegari, Coxeter groups and random groups, preprint (2015) arXiv: $1411.1506 \mathrm{v} 2$

[7] F R Cohen, T J Lada, J P May, The homology of iterated loop spaces, Lecture Notes in Mathematics 533, Springer, Berlin (1976) MR0436146

[8] M W Davis, The geometry and topology of Coxeter groups, London Mathematical Society Monographs Series 32, Princeton Univ. Press, NJ (2008) MR2360474

[9] F D Farmer, Cellular homology for posets, Math. Japon. 23 (1979) 607-613 MR529895

[10] S Galatius, O Randal-Williams, Homological stability for moduli spaces of high dimensional manifolds, I, preprint (2014) arXiv:1403.2334v1 
[11] J L Harer, Stability of the homology of the mapping class groups of orientable surfaces, Ann. of Math. 121 (1985) 215-249 MR786348

[12] A Hatcher, Homological stability for automorphism groups of free groups, Comment. Math. Helv. 70 (1995) 39-62 MR1314940

[13] A Hatcher, Algebraic topology, Cambridge Univ. Press (2002) MR1867354

[14] A Hatcher, K Vogtmann, Homology stability for outer automorphism groups of free groups, Algebr. Geom. Topol. 4 (2004) 1253-1272 MR2113904

[15] A Hatcher, N Wahl, Stabilization for mapping class groups of 3-manifolds, Duke Math. J. 155 (2010) 205-269 MR2736166

[16] R B Howlett, On the Schur multipliers of Coxeter groups, J. London Math. Soc. 38 (1988) 263-276 MR966298

[17] S-i Ihara, T Yokonuma, On the second cohomology groups (Schur-multipliers) of finite reflection groups, J. Fac. Sci. Univ. Tokyo Sect. I 11 (1965) 155-171 MR0190232

[18] T Januszkiewicz, J Świątkowski, Hyperbolic Coxeter groups of large dimension, Comment. Math. Helv. 78 (2003) 555-583 MR1998394

[19] M C Kerz, The complex of words and Nakaoka stability, Homology Homotopy Appl. 7 (2005) 77-85 MR2155519

[20] W Lück, Survey on classifying spaces for families of subgroups, from "Infinite groups: geometric, combinatorial and dynamical aspects" (L Bartholdi, T CecceheriniSilberstein, T Smirnova-Nagnibeda, A Zuk, editors), Progr. Math. 248, Birkhäuser, Basel (2005) 269-322 MR2195456

[21] H Maazen, Homology stability for the general linear group, $\mathrm{PhD}$ thesis, Utrecht University (1979) Available at http://tinyurl.com/jangjee

[22] G Moussong, Hyperbolic Coxeter groups, $\mathrm{PhD}$ thesis, Ohio State University (1988) Available at http://search.proquest.com/docview/303686972

[23] M Nakaoka, Decomposition theorem for homology groups of symmetric groups, Ann. of Math. 71 (1960) 16-42 MR0112134

[24] D Quillen, Finite generation of the groups $K_{i}$ of rings of algebraic integers, from "Algebraic $K$-theory, I: Higher $K$-theories" (H Bass, editor), Lecture Notes in Math. 341, Springer, Berlin (1973) 179-198 MR0349812

[25] O Randal-Williams, Homological stability for unordered configuration spaces, Q. J. Math. 64 (2013) 303-326 MR3032101

[26] L Solomon, A Mackey formula in the group ring of a Coxeter group, J. Algebra 41 (1976) 255-264 MR0444756

[27] J A Swenson, The mod-2 cohomology of finite Coxeter groups, $\mathrm{PhD}$ thesis, University of Minnesota (2006) MR2709085 Available at http://tinyurl.com/hknbr7a 
[28] N Wahl, Homological stability for automorphism groups, preprint (2014) arXiv: $1409.3541 \mathrm{v} 1$

Institute of Mathematics, University of Aberdeen

Aberdeen AB24 3UE, United Kingdom

r.hepworth@abdn.ac.uk

http://www.abdn.ac.uk/ncs/profiles/r.hepworth/

Received: 19 February 2015 Revised: 23 December 2015 
